九州大学学術情報リポジトリ

Kyushu University Institutional Repository

\title{
Recursive structures in the multispecies TASEP
}

Arita, Chikashi

Faculty of Mathematics, Kyushu University

Ayyer, Arvind

University of California Davis

Mallick, Kirone

Institut de Physique Théorique CEA

Sylvain, Prolhac

Zentrum Mathematik, Technische Universität München

http://hdl. handle. net/2324/19464

出版情報 : MI Preprint Series. 2011-9, 2011-03-31. 九州大学大学院数理学研究院 バージョン：

権利関係 : 


\title{
MI Preprint Series
}

Kyushu University

The Global COE Program

Math-for-Industry Education \& Research Hub

\section{Recursive structures in the multispecies TASEP}

\author{
Chikashi Arita \\ Arvind Ayyer, Kirone Mallick \& \\ Sylvain Prolhac
}

MI 2011-9

( Received March 31, 2011 )

Faculty of Mathematics

Kyushu University

Fukuoka, JAPAN 


\title{
Recursive structures in the multispecies TASEP
}

\author{
Chikashi Arita ${ }^{1}$, Arvind Ayyer ${ }^{2,3}$, \\ Kirone Mallick ${ }^{3}$, Sylvain Prolhac ${ }^{4}$ \\ ${ }^{1}$ Faculty of Mathematics, Kyushu University, Fukuoka 819-0395, Japan \\ 2 University of California Davis, One Shields Avenue, Davis 95616 USA \\ ${ }^{3}$ Institut de Physique Théorique CEA, F-91191 Gif-sur-Yvette, France \\ ${ }^{4}$ Zentrum Mathematik, Technische Universität München, Germany \\ E-mail: arita@math.kyushu-u.ac.jp ${ }^{1}$, ayyer@math.ucdavis.edu ${ }^{2}$, \\ kirone.mallick@cea.fr ${ }^{3}$, prolhac@ma.tum.de ${ }^{4}$
}

\begin{abstract}
We consider a multi-species generalization of the totally asymmetric simple exclusion process (TASEP) with the simple hopping rule: for $\alpha$ and $\beta$ thclass particles $(\alpha<\beta)$, the transition $\alpha \beta \rightarrow \beta \alpha$ occurs with a rate independent from the values $\alpha$ and $\beta$. P. A. Ferrari and J. Martin (2007) obtained the stationary state of this model thanks to a combinatorial algorithm, which was subsequently interpreted as a matrix product representation by Evans et al. (2009). This 'matrix ansatz' shows that the stationary state of the multi-species TASEP with $N$ classes of particles ( $N$-TASEP) can be constructed algebraically by the action of an operator on the $(N-1)$-TASEP stationary state. Besides, Arita et al. analyzed the spectral structure of the Markov matrix: they showed that the set of eigenvalues of the $N$-TASEP contains those of the $(N-1)$-TASEP and that the various spectral inclusions can be encoded in a hierarchical settheoretic structure known as the Hasse diagram. Inspired by these works, we define nontrivial operators that allow us to construct eigenvectors of the $\mathrm{N}$ TASEP by lifting the eigenvectors of the $(N-1)$-TASEP. This goal is achieved by generalizing the matrix product representation and the Ferrari-Martin algorithm. In particular, we show that the matrix ansatz is not only a convenient tool to write the stationary state but in fact intertwines Markov matrices of different values of $N$.
\end{abstract}




\section{Introduction}

Interacting particle systems are mathematical models used to study collective properties of N-body systems evolving with time [45]. Replacing the actual evolution rules (given by a classical or a quantum Hamiltonian) by a stochastic dynamics leads one to formulate many properties of the system in terms of probability theory and allows the use of powerful mathematical methods [34]. Hence, universal properties of nonequilibrium statistical physics are successfully explored through the investigation of stochastic processes, that are governed by simple dynamical rules at the microscopic level but that display a rich macroscopic behavior [41, 43]. When defining such models, some specific features of the original physical problem have to be discarded, but if the relevant and fundamental characteristics are retained (such as symmetry properties and conservation laws) it is expected that the large scale and large time behavior of the system will be described correctly [25]. Building a simple representation for complex phenomena is common procedure in statistical physics, leading to the emergence of paradigmatic models: the harmonic oscillator, the random walker, the Ising magnet and so on. These "beautiful models" often display wonderful mathematical structures $[9,47]$.

In the field of nonequilibrium statistical mechanics, the asymmetric simple exclusion process (ASEP) has reached the status of a paradigm [14, 29, 35]. The ASEP is a lattice-gas model of interacting particles. Each of these particles is a random walker that hops from a site to one of the neighboring locations, but a move is allowed only if the target site is empty. This exclusion constraint mimics short-range interactions amongst particles. In order to drive this lattice gas out of equilibrium, non-vanishing currents have to be established in the system and this can be achieved by various means: by starting with a non-uniform initial condition that takes an infinite amount of time to relax, by coupling the system to external reservoirs that drive currents through the system (transport of particles, energy, heat, etc.) or by introducing some intrinsic bias in the dynamics that favors motion in a privileged direction, each particle being an asymmetric random walker that drifts steadily along the direction of an external driving force. In particular, the case where moves are allowed in only one direction is said totally asymmetric (TASEP).

The ASEP has been invented several times and in different contexts due to its simplicity. It was probably first proposed as a prototype to describe the dynamics of ribosomes along RNA [36]. In the mathematical literature, Brownian motions with hard-core interactions were first studied by Spitzer [44] who coined the name exclusion process. The ASEP also appeared naturally in the description of transport processes in systems with strong geometric constraints such as macromolecules transiting through capillary vessels and that cannot overtake each other [32], or anisotropic conductors known as solid electrolytes where electrons hop from a vacant location to another and repel each other via Coulomb interaction [13]. Popular modern applications of the exclusion process include molecular motors that transport proteins within the cells along actin filaments [30] and, last but not least, the ASEP and its variants are ubiquitous in discrete models of traffic flow [40].

From the mathematical point of view, the ASEP is one of the simplest but nontrivial models for which the hydrodynamic limit can be rigorously proved. At large scales, the distribution of the particles of the ASEP emerges as a density field that evolves according to the Burgers equation with a vanishingly small viscosity $[31,45,48]$. The Burgers equation is the textbook prototype for shock formation: a 
smooth initial distribution can develop a singularity (a discontinuity) in finite time. A natural question that arises is whether this shock is an artifact of the hydrodynamic limit or if, under some specific conditions, the original ASEP does display some singularity at the microscopic scale $[3,4,11,12,27]$. This question was answered positively [16]: a shock does exist at the level of the particle system and its width is of the order of the lattice size. However, defining precisely the position of the shock at the microscopic level requires a trick which is achieved by introducing a new type of particle called a second-class particle, which amounts to coupling two TASEP models which initially differ at a single location [33]. This second-class particle, denoted by 2 , has the same dynamics as a normal (or first-class) particle, denoted by 1 , but firstclass particles treat it as a hole (denoted by 0 ). The local dynamical rules thus take the following simple form:

$$
10 \rightarrow 01 \quad 12 \rightarrow 21 \text { and } 20 \rightarrow 02,
$$

where all transitions occur with a same rate. It was proved rigorously in $[20,21]$ that the dynamics of the unique second-class particle mimics the motion of the shock in an infinite system. Alternatively, by considering a finite density of second-class particles in a periodic system, the shape of the microscopic fronts were exactly calculated [16].

A straightforward generalization of the two species case of first- and second-class particles is to the multispecies exclusion process where there is a hierarchy amongst $N$ different species (or classes) of particles [1, 2, 37] ( $N$-ASEP): a particle of class $\alpha$ views particles of classes $\beta>\alpha$ as holes and is viewed as a hole by particles of classes $\beta<\alpha$; in other words, "higher" classes have lower overtaking priority, as will be defined precisely in the next section $\ddagger$. The stationary state of this simplest multispecies generalization of the ASEP is highly nontrivial: it was first constructed by Pablo Ferrari and James Martin $[23,24]$ in the TASEP case through a mapping to queueing processes. Their construction was inspired by earlier combinatorial results $[5,22,17]$. In $[18,38]$, the Ferrari-Martin algorithm was re-expressed as a matrix product representation in which the weight of a configuration in the $N$-ASEP is written as a linear combination of weights of configurations of the $(N-1)$-ASEP. This matrix (product) ansatz hence allows one to derive steady-state properties of a given model knowing those of a simpler system.

More general spectral properties of the multispecies ASEP on a ring were investigated in [6] where models with different total number of species were related to each other in a different manner. In that work, the key observation was that particles of two consecutive classes cannot be distinguished by particles of other classes. Thus, identifying two consecutive classes $n$ and $n+1$ defines a natural projection from the $N$ ASEP onto the $(N-1)$-ASEP. Because one can identify any two consecutive classes of particles, there are $N$ different such projections. These mappings together with their commutation relations endow the set of all possible models with a poset structure represented by a Hasse diagram [6]. The existence of this structure leads to canonical inclusion relations and duality in the spectrum of the Markov matrix. In particular, through the identification mapping, the eigenvectors of the $N$-ASEP either vanish or project onto eigenvectors of the $(N-1)$-ASEP. However, these projections blur some essential information (namely, the difference between the two consecutive classes $n$ and $n+1)$ that cannot be retrieved easily. In particular, they do not allow one to build the eigenvectors of a model knowing those of a simpler system.

$\ddagger$ We mention here that multispecies ASEPs with other local-interaction rules have been introduced, see $[7,10,19,28]$ 
The above descriptions of the matrix ansatz and of the identification maps indicate that they operate in reverse directions and, hence, that they should be related. This also suggests that the matrix ansatz may have a range of applicability that exceeds stationary-state properties; the matrix ansatz should be useful also to build "excited states" of the $N$-ASEP knowing those of the $(N-1)$-ASEP. In this perspective, the matrix ansatz could be viewed as a 'lifting procedure' from the $(N-1)$-ASEP to the $N$-ASEP that creates a new species of particles by separating a given class into two consecutive subclasses. Further, we have seen that there are $N$ different choices for the identification maps from the $N$-ASEP to the $(N-1)$-ASEP. Are there also $N$ different matrix product representations that would correspond to splitting a species $n$ into two consecutive classes $n$ and $n+1$ ? Could there be $N$ different generalizations of the Ferrari-Martin algorithm?

The objective of the present work is to answer these questions in the TASEP case by reformulating them in the appropriate mathematical framework and stating them in rigorous terms. We will introduce a generalized matrix ansatz which constructs a lifting operator from the $(N-1)$-TASEP to the $N$-TASEP. This will provide with a new and much broader perspective about this technique and shed light on the recursive structures that underlie the $N$-TASEP dynamics. As a result, the problems stated above will be solved and families of quadratic algebras that encode these recursions will be constructed. The outline of this work is as follows. In section 2, we define the dynamical rules of the $N$-ASEP, describe the characteristics and some spectral properties of the Markov matrix, define identification operators between different systems (sectors) and formulate the fundamental problem addressed here. From section 3 onwards, we restrict our consideration to the TASEP case, unless explicitly stated otherwise. In section 3, we recall the Ferrari-Martin algorithm of the stationary state of the $N$-TASEP and describe the associated matrix product representation. The main results are derived in section 4: we construct a set of generalized matrix ansatz and we prove that this allows us to define a family of conjugation operators from the $(N-1)$-TASEP to the $N$-TASEP; each of these matrix ansatz leads to a different quadratic algebra and we find explicit representations for all these algebras; finally, we find a generalized Ferrari-Martin algorithm corresponding to each algebra. Concluding remarks are given in section 5. The appendices contains examples and technical details.

\section{The $N$-ASEP: Definition and properties}

\subsection{Definition of the model}

The $N$-ASEP on the ring $\mathbb{Z}_{L}$ with $L$ sites is defined by the following dynamical rules. Each site $i \in \mathbb{Z}_{L}$, is assigned with a variable (local state) $k_{i} \in\{1, \ldots, N+1\}(N \geq 0)$. We introduce a stochastic process such that nearest neighbor pairs of local states $(\alpha, \beta)=\left(k_{i}, k_{i+1}\right)$ are interchanged with the following transition rates:

$$
\alpha \beta \rightarrow \beta \alpha \begin{cases}p & \text { if } \alpha<\beta, \\ q & \text { if } \alpha>\beta,\end{cases}
$$

$p$ and $q$ being real nonnegative parameters, with the choice $q \leq p$ without loss of generality $\S$. In particular, for $q=0$ the model is totally asymmetric and is called the

$\S$ In the paper [6], the dynamical rule is defined as $\left\{\begin{array}{ll}p & \text { if } \alpha>\beta, \\ q & \text { if } \alpha<\beta .\end{array}\right.$ We have to replace $p \leftrightarrow q$ or reverse the variables $k_{i} \rightarrow N+2-k_{i}$, if we wish to compare the present work with [6]. 
$N$-TASEP; for $0<q<p$, the model is said partially asymmetric ( $N$-PASEP); $q=p$ is the symmetric case $(N$-SSEP).

The dynamics is formulated in terms of the continuous-time master equation for the probability $P\left(k_{1} \cdots k_{L} ; t\right)$ of finding a configuration $k_{1} \cdots k_{L}$ at time $t$ :

$$
\begin{aligned}
\frac{d}{d t} P\left(k_{1} \cdots k_{L} ; t\right)= & \sum_{i \in \mathbb{Z}_{L}} \Theta\left(k_{i+1}-k_{i}\right) P\left(k_{1} \cdots k_{i-1} k_{i+1} k_{i} k_{i+2} \cdots k_{L} ; t\right) \\
& -\sum_{i \in \mathbb{Z}_{L}} \Theta\left(k_{i}-k_{i+1}\right) P\left(k_{1} \cdots k_{L} ; t\right),
\end{aligned}
$$

where $\Theta$ is a step function defined as

$$
\Theta(x)= \begin{cases}p & (x<0) \\ 0 & (x=0) \\ q & (x>0)\end{cases}
$$

This model can be regarded as an interacting multispecies particle system by interpreting the local state $k_{i}=\alpha$ as representing the site $i$ occupied by a particle of the $\alpha$ th kind. In the conventional terminology, a particle of the $\alpha$ th kind for $1 \leq \alpha \leq N$ is called " $\alpha$ th-class particle." We can view the local state $N+1$ as a vacant site (or a hole), which is often denoted by 0 . However, for later convenience, it is better to use $N+1$ instead of 0 and we shall stick to that convention.

\subsection{The Markov matrix}

Let $\{|1\rangle, \ldots,|N+1\rangle\}$ be the basis of the single-site space $\mathbb{C}^{N+1}$. The tensor product $\left|k_{1} \cdots k_{L}\right\rangle=\left|k_{1}\right\rangle \otimes \cdots \otimes\left|k_{L}\right\rangle \in\left(\mathbb{C}^{N+1}\right)^{\otimes L}$ corresponds to the configuration $k_{1} \cdots k_{L}$ on the ring. The probability vector at time $t$ can be written as

$$
|P(t)\rangle=\sum_{1 \leq k_{i} \leq N+1} P\left(k_{1} \cdots k_{L} ; t\right)\left|k_{1} \cdots k_{L}\right\rangle .
$$

In this language, the master equation (3) becomes

$$
\frac{d}{d t}|P(t)\rangle=M^{(N)}|P(t)\rangle
$$

where the (total) Markov matrix $M^{(N)}$ is of size $(N+1)^{L}$ by $(N+1)^{L}$. This Markov matrix can be written as the sum of local linear operators $\left(M_{\mathrm{Loc}}^{(N)}\right)_{i, i+1}$ acting only on the $i$ th and the $(i+1)$ th components of the tensor product:

$$
M^{(N)}=\sum_{i \in \mathbb{Z}_{L}}\left(M_{\text {Loc }}^{(N)}\right)_{i, i+1},
$$

where the action of the local Markov matrices on a bond is given by

$$
M_{\text {Loc }}^{(N)}=\sum_{\alpha, \beta=1}^{N+1}(-\Theta(\alpha-\beta)|\alpha \beta\rangle\langle\alpha \beta|+\Theta(\alpha-\beta)| \beta \alpha\rangle\langle\alpha \beta|) .
$$

Note that off-diagonal elements of $M^{(N)}$ are $p, q$ or 0 , and the diagonal elements are expressed as $p x+q y$ with nonpositive integers $x$ and $y$. The sum of entries in each column of $M^{(N)}$ is 0 , assuring the conservation of the total probability $\sum_{1 \leq k_{i} \leq N+1} P\left(k_{1} \cdots k_{L} ; t\right)$. The master equation can be solved formally as

$$
|P(t)\rangle=\mathrm{e}^{t M^{(N)}}|P(0)\rangle
$$


and thus the eigenvalues and the right eigenvectors of $M^{(N)}$ give information for physical properties of the model. The Markov matrix has the eigenvalue 0 , and we call the corresponding right eigenvectors stationary states. All the other eigenvalues have strictly negative real parts (Perron-Frobenius theorem [45]), which characterize the relaxation to the stationary states. We remark that the Markov matrix $M^{(N)}$ is Hermitian only for $q=p$, where it becomes the Hamiltonian of the $s l(N+1)$-invariant Heisenberg spin chain.

\subsection{Particle conservation and Hasse diagram structure}

In view of the transition rule (2), the total Markov matrix obviously preserves the number of particles of each kind. Let $m_{\alpha} \geq 0$ denote the number of particles of the $\alpha$ th class (respectively holes), for $1 \leq \alpha \leq N$ (respectively $\alpha=N+1$ ):

$$
\underbrace{1 \cdots 1}_{m_{1}} \underbrace{2 \cdots 2}_{m_{2}} \cdots \underbrace{N+1 \cdots N+1}_{m_{N+1}} .
$$

Then, the state space decomposes into sectors labeled by $m=\left(m_{1}, \ldots, m_{N+1}\right)$ with the constraint $m_{1}+\cdots+m_{N+1}=L$ :

$$
\left(\mathbb{C}^{N+1}\right)^{\otimes L}=\bigoplus_{m} V_{m}
$$

The Markov matrix has a block diagonal structure, leaving each sector invariant

$$
M^{(N)}=\bigoplus_{m} M_{m}, \quad M_{m} \in \operatorname{End} V_{m},
$$

where the square matrix $M_{m}$ acts on the vector space $V_{m}$ spanned by all configurations belonging to the sector $m$ :

$$
V_{m}=\bigoplus_{\#\left\{i \mid k_{i}=j\right\}=m_{j}} \mathbb{C}\left|k_{1} \cdots k_{L}\right\rangle .
$$

The dimension of $V_{m}$ is given by $\operatorname{dim} V_{m}=\frac{L !}{m_{1} ! \cdots m_{N+1} !}$ and the total number of sectors for given $L$ and $N$ is $\left(\begin{array}{c}L+N \\ N\end{array}\right)$.

Let us call a sector $m=\left(m_{1}, \ldots, m_{N+1}\right)$ a basic sector if $m_{n}>0$ for all $n=1, \ldots, N+1$ (i.e. there exists at least one particle of each type). Note that there are no basic sectors for $N \geq L$. Thus, we shall always take $N \leq L-1$. For example, for $L=4$, the list of all basic sectors for different values of $N$ is given by

$$
\begin{array}{cc}
N=3: & (1,1,1,1), \\
N=2: & (2,1,1),(1,2,1),(1,1,2), \\
N=1: & (3,1),(2,2),(1,3), \\
N=0: & (4) .
\end{array}
$$

The number of basic sectors for given $L$ and $N$ is given by $\left(\begin{array}{c}L-1 \\ N\end{array}\right)$. Let $\mathcal{M}$ be the set of all labels for the basic sectors:

$$
\mathcal{M}=\left\{\left(m_{1}, \ldots, m_{N+1}\right) \mid 0 \leq N \leq L-1, m_{i} \in \mathbb{N}, m_{1}+\cdots+m_{N+1}=L\right\} .
$$

We now introduce an alternative labeling of the basic sectors [6] that will be very useful in the following: let $s_{j}$ be the total number of particles of classes $k \leq j$, i.e.

$$
s_{j}=m_{1}+m_{2}+\cdots+m_{j} .
$$


We have $m_{j}=s_{j}-s_{j-1}>0$ with the convention $s_{0}=0$, and thus each basic sector of the $N$-ASEP can be labeled by the set $\mathfrak{s}=\left\{s_{1}, \ldots, s_{N}\right\}$ with $0<s_{1}<s_{2}<\cdots<$ $s_{N}<L$. The set $\mathfrak{s}$ is a subset of $\Omega=\{1,2, \ldots, L-1\}$, i.e. the set $\mathfrak{s}$ is an element of $\mathcal{S}$, the power set (the set of all subsets) of $\Omega$. We can identify $\mathcal{M}$ with $\mathcal{S}$ by the oneto-one correspondence (16). In the following, we shall use both labels equivalently: for instance, the invariant vector spaces (respectively the Markov matrices acting on them) will be denoted either by $V_{m}$ or $V_{\mathfrak{s}}$ (respectively $M_{m}$ or $M_{\mathfrak{s}}$ ).

An example of the identification $\mathcal{M} \leftrightarrow \mathcal{S}$ for $L=4$ is given in figure 1 . The set $\mathcal{S}$ is equipped with a natural poset (partially ordered set) structure with respect to the inclusion $\subseteq$, which is encoded in the Hasse diagram [46]. In the present case, it is just the $L-1$ dimensional hypercube, where each vertex corresponds to a sector. Every link of the hypercube becomes an arrow $\mathfrak{t} \rightarrow \mathfrak{s}$ meaning that $\mathfrak{t} \subset \mathfrak{s}$ and $\# \mathfrak{s}=\# \mathfrak{t}+1$. The "maximal sector" $\Omega=\{1,2, \ldots, L-1\}$ corresponds to a unique sink and the "minimal sector" $\emptyset$ corresponds to a unique source, as in figure 1 .

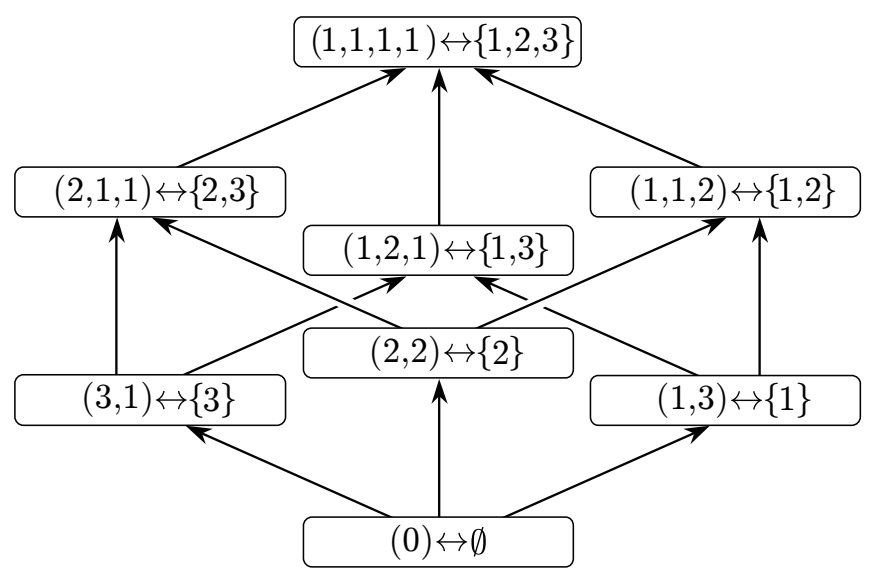

Figure 1. Basic sectors for $L=4$ in the Hasse diagram. Each sector is labeled by an element of $\mathcal{M}$ and one of $\mathcal{S}$.

The following spectral properties in this Hasse diagram were proved in [6]:

- Spectral inclusion: Let $\operatorname{Spec}(\mathfrak{s})$ be the multiset of the eigenvalues of $M_{\mathfrak{s}}$ with the multiplicity of an element representing the degree of its degeneracy. Then,

$$
\operatorname{Spec}(\mathfrak{s}) \supset \operatorname{Spec}(\mathfrak{t})
$$

for any pair of sectors $\mathfrak{s} \supset \mathfrak{t}$. In particular, $\operatorname{Spec}(\Omega)$ contains the eigenvalues of the Markov matrix $M_{\mathfrak{s}}$ of all the sectors $\mathfrak{s} \in \mathcal{S}$. An example of this spectral inclusion for $L=4$ is displayed in figure 2 .

- Spectral duality: Using the spectral inclusion theorem, we can classify the eigenvalues $E \in \operatorname{Spec}(\mathfrak{s})$ into two types: (i) eigenvalues that already exist in lower sectors $\mathfrak{u} \subset \mathfrak{s}$, (ii) eigenvalues that appear at $\mathfrak{s}$. Let us call eigenvalues of the second type genuine eigenvalues. More explicitly, the genuine spectrum $\operatorname{Spec}^{\circ}(\mathfrak{s})$ of the sector $\mathfrak{s}$ is defined as

$$
\operatorname{Spec}^{\circ}(\emptyset):=\operatorname{Spec}(\emptyset)=\{0\}, \quad \operatorname{Spec}^{\circ}(\mathfrak{s}):=\operatorname{Spec}(\mathfrak{s}) \backslash \bigcup_{\mathfrak{u} \subset \mathfrak{s}} \operatorname{Spec}(\mathfrak{u}) .
$$




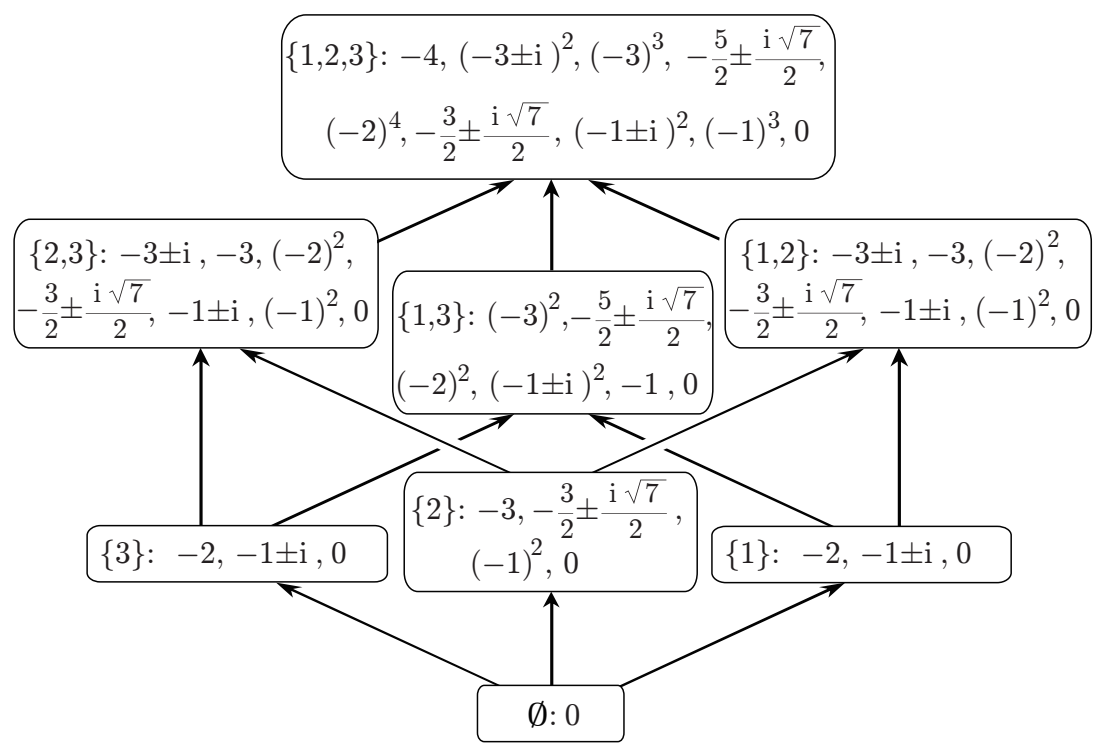

Figure 2. $\operatorname{Spec}(\mathfrak{s})$ for $L=4$ with $p=1$ and $q=0$ (TASEP case). Each superscript denotes the multiplicity. For example, the Markov matrix $M_{\{1,3\}}$ has eigenvalue -2 , and its degree of degeneracy is 2 .

Then, the following duality relation was proved:

$$
\operatorname{Spec}^{\circ}(\mathfrak{s})=-L(p+q)-\operatorname{Spec}^{\circ}(\overline{\mathfrak{s}}),
$$

where the sector $\overline{\mathfrak{s}}=\Omega \backslash \mathfrak{s}$ is furthest from the sector $\mathfrak{s}$ in the Hasse diagram.

These two spectral relations were derived by introducing a family of linear operators connecting different sectors, that we now review.

\subsection{The identification operators $\varphi_{\mathrm{ts}}$ and the conjugation property}

Let us consider two (basic) sectors $\mathfrak{s}=\left\{s_{1}<\cdots<s_{N}\right\}$ and $\mathfrak{t}=\mathfrak{s} \backslash\left\{s_{n_{1}}, \ldots, s_{n_{u}}\right\}$. We introduce a linear operator $\varphi_{\mathfrak{t} \mathfrak{s}}: V_{\mathfrak{s}} \rightarrow V_{\mathfrak{t}}$, whose action on the basis vectors is given by

$$
\left|k_{1} \cdots k_{L}\right\rangle \in V_{\mathfrak{s}} \mapsto\left|k_{1}^{\prime} \cdots k_{L}^{\prime}\right\rangle \in V_{\mathfrak{t}}, \quad \text { with } x^{\prime}=x-\#\left\{i \mid n_{i}<x\right\} .
$$

Note that $\varphi_{\mathfrak{t} \mathfrak{s}}: V_{\mathfrak{s}} \rightarrow V_{\mathfrak{t}}$ is surjective. For $\mathfrak{s}=\mathfrak{t}, \varphi_{\mathfrak{s} \mathfrak{s}}$ is the identity operator for any sector $\mathfrak{s}$.

To understand the general definition (20), we give an example $\mathfrak{s}=\{2,3,5,8\} \supset$ $\mathfrak{t}=\{2,5\}$ with $L=9$. These two sectors correspond to the following compositions of the system into different types of particles:

$$
\begin{aligned}
& \text { s : }\left.\left.\left.\left.\quad 1 \quad 1\right|^{2} 2\right|^{3} 3 \quad 3\right|^{5} 4 \quad 4 \quad 4\right|^{8} 5 \\
& \mathfrak{t}: \quad 1 \quad 1 \mid \begin{array}{lll|llll}
2 & 2 & 2 & 3 & 3 & 3 & 3
\end{array}
\end{aligned}
$$


According to these lists, we define $\varphi_{\mathfrak{t s}}$ to be the operator replacing (or identifying) the local states according to the rules $3 \rightarrow 2,4 \rightarrow 3,5 \rightarrow 3$ (keeping 1 and 2 unchanged) within all the ket vectors $\left|k_{1} \cdots k_{L}\right\rangle$ in $V_{\mathfrak{s}}$. For example,

$$
\varphi_{\mathfrak{t s}}|345214431\rangle=|233213321\rangle \text {. }
$$

More generally, for a pair of sectors $\mathfrak{t} \subseteq \mathfrak{s}$, consider a chain $\mathfrak{s}_{0} \supset \mathfrak{s}_{1} \supset \cdots \supset \mathfrak{s}_{u}$ of sectors such that $\mathfrak{s}_{0}=\mathfrak{s}_{\mathfrak{s}_{u}}=\mathfrak{t}$ and $\# \mathfrak{s}_{j}=\# \mathfrak{s}_{j+1}+1$ for all $0 \leq j<u$. Then, the decomposition

$$
\varphi_{\mathfrak{t s}_{\mathfrak{s}}}=\varphi_{\mathfrak{t s}_{u-1}} \varphi_{\mathfrak{s}_{u-1} \mathfrak{s}_{u-2}} \cdots \varphi_{\mathfrak{s}_{2} \mathfrak{s}_{1}} \varphi_{\mathfrak{s}_{1} \mathfrak{s}}
$$

holds and is independent of the choice of the intermediate sectors [6].

\subsection{The conjugation property}

A crucial property of the identification operators is that they provide a conjugation between different sectors. More precisely, for any two sectors such that $\mathfrak{s} \supseteq \mathfrak{t}$ the following diagram commutes:

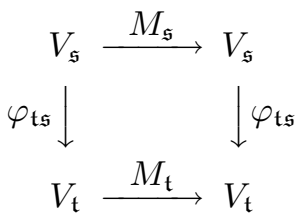

which means

$$
M_{\mathfrak{t}} \varphi_{\mathfrak{t s}}=\varphi_{\mathfrak{t} \mathfrak{s}} M_{\mathfrak{s}} .
$$

We shall call this type of relation a conjugation relation, relating the dynamics governed by $M_{\mathfrak{s}}$ and that governed by $M_{\mathrm{t}}$. The identification operator $\varphi_{\mathfrak{t s}}$ intertwines the two Markov matrices $M_{\mathfrak{s}}$ and $M_{\mathfrak{t}}$, and we call $\varphi_{\mathfrak{t s}}$ a conjugation matrix. The relation (25) was the key [6] in proving the spectral properties (17) and (19). Each right eigenvector $|E\rangle$ with eigenvalue $E$ in the sector $\mathfrak{s}$ can be projected down by $\varphi_{\mathfrak{t s}}$ to the sector $\mathfrak{t}$, and $\varphi_{\mathfrak{t} \mathfrak{s}}|E\rangle$ is also a right eigenvector with eigenvalue $E$ in the sector $\mathfrak{s}$ (under the assumption $\varphi_{\mathfrak{t s}}|E\rangle \neq 0$ ). In particular, the stationary state of a sector $\mathfrak{s}$ is mapped by the identification operator to the stationary state of any sector $\mathfrak{t}$ such that $\mathfrak{t} \subseteq \mathfrak{s}$.

\subsection{Looking for an inverse conjugation relation}

The identification operator $\varphi_{\mathfrak{t s}}$ always maps an upper (more complex) sector $\mathfrak{s}$ into a lower (simpler) sector $\mathfrak{t}$. This implies that identification matrix $\varphi_{\mathfrak{t} \mathfrak{s}}$ cannot help us to construct a right eigenvector in the sector $\mathfrak{s}$ knowing the eigenvector with the same eigenvalue in a smaller sector $\mathfrak{t}$. It would be very useful if we could lift information from lower sectors to upper sectors in the Hasse diagram. One of the motivations of the present work can be formulated as follows: Can we construct a matrix $\psi_{\mathfrak{s t}}$ which lifts up right eigenvectors from a lower sector $\mathfrak{t}$ to an upper sector $\mathfrak{s}$ ? This would be possible if we could define an operator $\psi_{\mathfrak{s t}}$ from $V_{\mathfrak{t}}$ to $V_{\mathfrak{s}}$ such that the following diagram commutes:

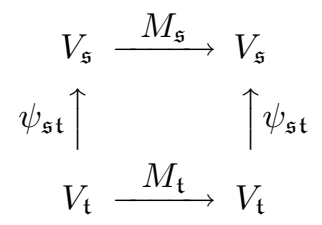


Equivalently, for any sectors $\mathfrak{t} \subseteq \mathfrak{s}$ the conjugation matrix $\psi_{\mathfrak{s t}}$ satisfies

$$
\psi_{\mathfrak{s t}} M_{\mathfrak{t}}=M_{\mathfrak{s}} \psi_{\mathfrak{s t}} .
$$

Note that the directions of the vertical arrows are opposite as compared to those in the previous diagram (24), which is a crucial difference. Equivalently, comparing equation (27) with equation (25), we observe that the order of the products is opposite. The property (27) has the following important consequence: Let $\left|E_{\mathrm{t}}\right\rangle$ be any eigenvector of $M_{\mathfrak{t}}$ with eigenvalue $E$. Then

$$
M_{\mathfrak{s}}\left(\psi_{\mathfrak{s t}}\left|E_{\mathfrak{t}}\right\rangle\right)=\psi_{\mathfrak{s t}}\left(M_{\mathfrak{t}}\left|E_{\mathfrak{t}}\right\rangle\right)=E\left(\psi_{\mathfrak{s t}}\left|E_{\mathfrak{t}}\right\rangle\right),
$$

which means that $\psi_{\mathfrak{s t}}\left|E_{\mathfrak{t}}\right\rangle=\left|E_{\mathfrak{s}}\right\rangle$ is an eigenvector of $M_{\mathfrak{s}}$ with the same eigenvalue $E$ (if $\psi_{\mathfrak{s t}}\left|E_{\mathfrak{t}}\right\rangle \neq 0$ ). In other words, the map $\psi_{\mathfrak{s t}}$ allows to lift eigenvectors from a smaller sector to a larger sector and provides a constructive information from a simpler system to a more complex one (whereas the identification operator $\varphi_{\mathfrak{t s}}$ erases information). The existence of $\psi_{\mathfrak{s t}}$ is a very nontrivial property of the model. In the next section, we review the construction of the stationary state, which will be generalized later to define $\psi_{\mathfrak{s t}}$.

\section{The stationary state of the $N$-TASEP}

From this section on, we set $p=1$ and $q=0$, i.e. we consider the TASEP case, unless explicitly stated otherwise. In the $N$-TASEP dynamics, each particle can hop to its right nearest-neighbor site if the target site is empty or occupied by a higher-class particle.

A stationary state is a right eigenvector of the Markov matrix corresponding to the eigenvalue 0 . We denote it by $|\bar{P}\rangle=\sum_{\tau} \bar{P}(\tau)|\tau\rangle$ :

$$
0=M^{(N)}|\bar{P}\rangle \text {. }
$$

For each sector $m$, the Markov matrix has a unique stationary state $\left|\bar{P}_{m}\right\rangle$ up to an overall constant factor. For basic sectors $\mathfrak{s} \leftrightarrow m$, we write $\left|\bar{P}_{\mathfrak{s}}\right\rangle=\left|\bar{P}_{m}\right\rangle$. From the "grand-canonical" stationary state $|\bar{P}\rangle$, which is a solution of equation (29), we extract the stationary state of each sector, by restricting the components of $|\bar{P}\rangle$ to that sector. We emphasize that the multispecies TASEP on a ring does not satisfy the detailed-balance condition and exhibits non-vanishing currents in its stationary state, which is one of the major characteristics of nonequilibrium systems $\|$.

The stationary state of the $N$-TASEP is nontrivial as soon as $N \geq 2$. The stationary state of the 2-TASEP was constructed by using a matrix product representation in [16]. However, this technique did not seem easily generalizable to higher values of $N$ (see [37] for an attempt for the case $N=3$ ). The solution to this problem came from two completely different directions. In [5] it was shown that the stationary state provided by the matrix product representation for the 2-TASEP could be interpreted in terms of weights in a binary tree. On the other hand, in [22], these weights were rewritten in terms of Dyck paths which also appear as trajectories of a queueing process and therefore the 2-TASEP was reinterpreted as a queueing process. This fact was generalized to the $N$-TASEP which was mapped into a system of coupled queueing processes [24]: this construction leads to the Ferrari-Martin algorithm for the stationary state of the $N$-TASEP that we review in the next subsection.

\| However, it is important to keep in mind that boundary conditions are absolutely crucial in nonequilibrium physics: indeed, in a closed segment with reflecting boundaries, the detailed-balance condition is satisfied [10] 


\subsection{Review of Ferrari and Martin's construction}

We now reformulate the algorithm found in [24] that constructs the stationary state of the $N$-TASEP, in terms of the convention adopted in the present work. The basic idea is to obtain the stationary state of $N$-TASEP from that of $(N-1)$-TASEP. The following algorithm is valid for any sector $\mathfrak{s}=\left\{s_{1}<\cdots<s_{N}\right\}$; In figure 3 , we provide an explicit example for a ring of size $L=9$ and with $\mathfrak{s}=\{2,5,6\}$ (i.e. $m_{1}=2, m_{2}=3, m_{3}=1$ and $\left.m_{4}=3\right)$.
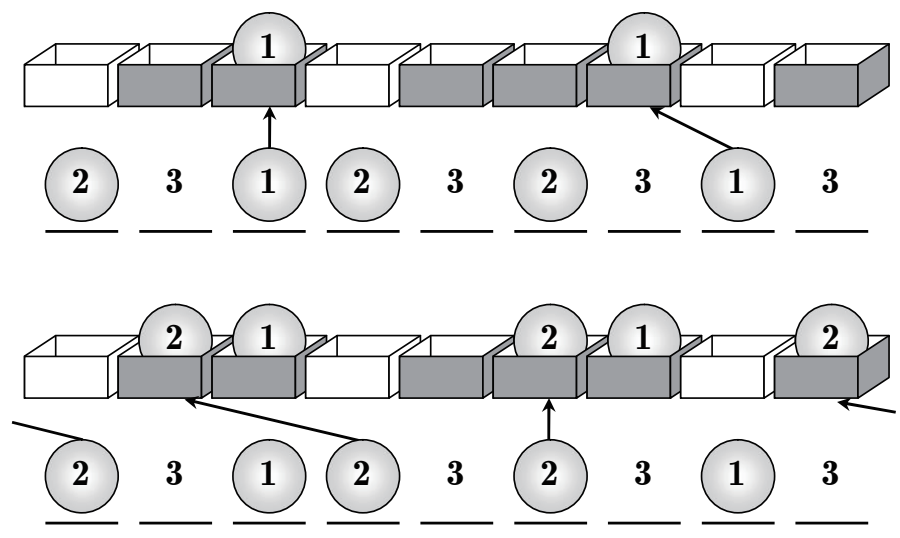

(iii) (iv)

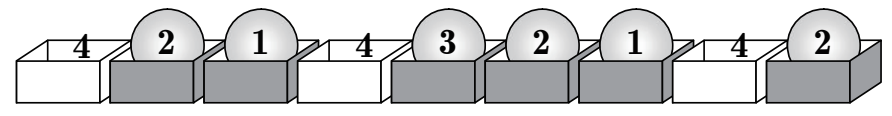

Figure 3. The Ferrari-Martin algorithm that constructs a configuration of $\mathrm{N}$ TASEP from that of $(N-1)$-TASEP. This figure provides the specific example: $F(w b b w b b b w b, 231232313)=421432142$.

In order to relate a configuration of the $(N-1)$-TASEP to a configuration of the $N$-TASEP, we consider two lines, each of them corresponding to a lattice of size $L$.

(i) On the upper line, we set $s_{N}$ black boxes $b$ and $\left(L-s_{N}\right)$ white boxes $w$ arbitrarily, that we write as $c_{1} \cdots c_{L}$ (with $c_{i}=b, w$ ).

On the lower line, there is a configuration $k_{1} \cdots k_{L}$ of the $(N-1)$-TASEP corresponding to the sector $\mathfrak{s} \backslash\left\{s_{N}\right\}$ : thus, on the lower line, there are $\left(s_{\nu}-s_{\nu-1}\right)$ $\nu$ th-class particles $\left(1 \leq \nu \leq N-1, s_{0}=0\right)$ and $\left(L-s_{N-1}\right) N$ th-class particles (i.e. empty sites).

(ii-1) Let $\left\{i_{1}^{(1)}, \ldots, i_{s_{1}}^{(1)}\right\}$ be the positions of the $s_{1}$ first-class particles on the lower line. For the first first-class particle located at $i_{1}^{(1)}$, find the nearest black box $c_{i^{\prime}}=b$ with $i^{\prime} \leq i_{1}^{(1)}$ and put a particle of type 1 on it. If there is no such black box, put the particle 1 on the rightmost black box. For the second first-class particle located at $i_{2}^{(1)}$, find the nearest unoccupied black box $c_{i^{\prime}}=b$ with $i^{\prime} \leq i_{2}^{(1)}$ and put a particle of type 1 on it. If there is no such black box, put the particle of type 1 on the rightmost unoccupied black box. Iterate this procedure $s_{1}$ times: i.e., 
find the nearest unoccupied black box $c_{i^{\prime}}=b$ with $i^{\prime} \leq i_{\ell}^{(1)}$ for the $\ell$ th first-class particle located at $i_{\ell}^{(1)}$, and put a particle of type 1 on it or on the rightmost unoccupied black box if $i^{\prime}$ does not exist.

(ii-2) Now we consider the second-class particles on the lower line. Recall that there are $m_{2}=s_{2}-s_{1}$ of them, and set their positions as $\left\{i_{1}^{(2)}, \ldots, i_{s_{2}-s_{1}}^{(2)}\right\}$. There are $\left(s_{N}-s_{1}\right)$ unoccupied black boxes remaining on the upper line. We must iterate the following procedure $\left(s_{2}-s_{1}\right)$ times: find the nearest unoccupied black box $c_{i^{\prime}}=b$ with $i^{\prime} \leq i_{\ell}^{(2)}$ for the $\ell$ th second-class particle $\left(1 \leq \ell \leq s_{2}-s_{1}\right)$, and put a particle of type 2 on it or on the rightmost unoccupied black box if $i^{\prime}$ does not exist.

(ii- $\nu)$ In the same way, we go on for third-, fourth-, $\cdots,(N-1)$ th-class particles: for the $\nu$ th-class particles, there are $\left(s_{\nu}-s_{\nu-1}\right) \nu$ th-class particles on the lower line, with positions $\left\{i_{1}^{(\nu)}, \ldots, i_{s_{\nu}-s_{\nu-1}}^{(\nu)}\right\}$. There are $\left(s_{N}-s_{\nu-1}\right)$ unoccupied black boxes remaining on the upper line. Iterate the following procedure $\left(s_{\nu}-s_{\nu-1}\right)$ times: find the nearest unoccupied black box $c_{i^{\prime}}=b$ with $i^{\prime} \leq i_{\ell}^{(\nu)}$ for the $\ell$ th $\nu$ th-class particle $\left(1 \leq \ell \leq s_{\nu}-s_{\nu-1}\right)$, and put a particle of type $\nu$ on it or on the rightmost unoccupied black box if $i^{\prime}$ does not exist.

(iii) Now, there are $\left(s_{N}-s_{N-1}\right)$ unoccupied black boxes that remain. Put particles of type $N$ on them.

(iv) Regarding the $\left(L-s_{N}\right)$ white boxes as an empty sites, i.e. as particles of type $N+1$, we have thus constructed a well-defined configuration $F\left(c_{1} \cdots c_{L}, k_{1} \cdots k_{L}\right)$ of the $N$-TASEP on the upper line, belonging to the sector $\mathfrak{s}$, starting from a configuration $k_{1} \cdots k_{L}$ of the $(N-1)$-TASEP on the lower line that was in the sector $\mathfrak{s} \backslash\left\{s_{N}\right\}$. (Note that an $N$ represents an empty site in the sector $\mathfrak{s} \backslash\left\{s_{N}\right\}$ whereas $N+1$ does in $\mathfrak{s}$.)

Note that different configurations of $(N-1)$-TASEP on the lower line can lead to a same configuration of the $N$-TASEP on the upper line: for example,

$$
F(b b w w b, 32133)=F(b b w w b, 31323)=21443 .
$$

It was proved in [24] that the stationary weight of a given configuration in the sector $\mathfrak{s}$ is given (up to normalization) by the sum of the weights of all configurations in $\mathfrak{s} \backslash\left\{s_{N}\right\}$ that are related to it through this construction. Equivalently, we have

$$
\left|\bar{P}_{\mathfrak{s}}\right\rangle=\sum\left|F\left(c_{1} \cdots c_{L}, k_{1} \cdots k_{L}\right)\right\rangle\left\langle k_{1} \cdots k_{L} \mid \bar{P}_{\mathfrak{s} \backslash\left\{s_{N}\right\}}\right\rangle .
$$

Here the summation $\sum$ runs over $c_{1} \cdots c_{L}$ and $k_{1} \cdots k_{L}$ with $\#\left\{i \mid c_{i}=b\right\}=s_{N}$ and $k_{1} \cdots k_{L}$ belonging to the sector $\mathfrak{s} \backslash\left\{s_{N}\right\}$.

\subsection{Matrix product representation for the stationary state}

The Ferrari-Martin algorithm was restated as a matrix product representation in [18]. The basic idea of the matrix product representation is to express the stationary probability as the trace of a product of matrices over a suitable algebra. This technique, initially invented in [15] for the (one-species) ASEP with open boundaries, has been generalized to many stochastic interacting particle systems including discretetime updates, a second-nearest neighbor interaction and non-conservative dynamics 
(see [10] for an exhaustive review). For the $N$-TASEP on $\mathbb{Z}_{L}$, the stationary weight of a configuration $j_{1} \ldots j_{L}$ is given by the trace of an $L$-fold matrix product:

$$
\bar{P}\left(j_{1} \cdots j_{L}\right)=\operatorname{Tr}\left[X_{j_{1}}^{(N)} \cdots X_{j_{L}}^{(N)}\right]
$$

or equivalently as

$$
|\bar{P}\rangle=\operatorname{Tr}\left(\begin{array}{c}
X_{1}^{(N)} \\
\vdots \\
X_{N+1}^{(N)}
\end{array}\right)^{\otimes L}
$$

In [38], an explicit solution for the operators $X_{J}^{(N)}$ 's was found. It is given by the following tensor product recursions

$$
X_{J}^{(N)}=\sum_{K=1}^{N} a_{J K}^{(N, N)} \otimes X_{K}^{(N-1)} \quad \text { for } 1 \leq J \leq N+1
$$

with $Y \otimes X_{1}^{(1)}=Y \otimes X_{2}^{(1)}=Y$ (i.e., $X_{1}^{(1)}=X_{2}^{(1)}=1$ ). The operators $a_{J K}^{(N, N)}$ are given in the following table:

\begin{tabular}{|c|c|c|}
\hline$J \backslash K$ & $1 \cdots$ & $N-1$ \\
\hline $1 \cdots N-1$ & $\begin{array}{c}A^{\otimes(J-1)} \\
\otimes \delta \otimes \mathbb{1}^{\otimes(K-J-1)}\end{array}$ & $A^{\otimes(J-1)} \otimes \delta \otimes \mathbb{1}^{\otimes(N-J-1)}$ \\
\hline$N$ & 0 & $A^{\otimes(N-1)}$ \\
\hline$N+1$ & $\mathbb{1}^{\otimes(K-1)} \otimes \epsilon \otimes \mathbb{1}^{\otimes(N-K-1)}$ & $\mathbb{1}^{\otimes(N-1)}$ \\
\hline
\end{tabular}

We read $\delta \otimes \mathbb{1}^{\otimes(-1)} \otimes \epsilon=\mathbb{1}$ and $\delta \otimes \mathbb{1}^{\otimes x} \otimes \epsilon=0$ for $x \leq-2$. The operators $\delta, \epsilon$ and $A$ are the fundamental building blocks that are ubiquitous in the matrix ansatz technique [10]. These three operators generate a quadratic algebra and satisfy the following relations:

$$
\delta \epsilon=\mathbb{1}, \quad \delta A=0, \quad A \epsilon=0 .
$$

A common representation of this algebra is given by the infinite dimensional matrices

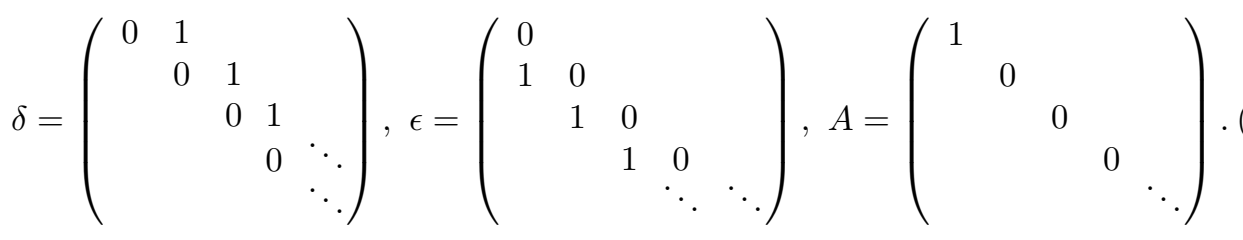

Remark: The Ferrari-Martin algorithm can not be easily defined for the PASEP because the directionality plays a crucial role in the algorithm. Nevertheless, the matrix product representation can readily be generalized to the PASEP case $(p=1$ and $q \neq 0$ ) as follows: in the table (35), we replace the operators $\delta, \epsilon$ and $A$ by the ' $q$ deformed' operators $\delta_{q}, \epsilon_{q}$ and $A_{q}$ that generate a quadratic algebra with the following relations

$$
\delta_{q} \epsilon_{q}-q \epsilon_{q} \delta_{q}=(1-q) \mathbb{1}, \quad \delta_{q} A_{q}=q A \delta_{q}, \quad A_{q} \epsilon_{q}=q \epsilon_{q} A_{q} .
$$

Then, the matrix product representation (33) with this deformation provides the stationary state of the $N$-PASEP, as was shown in [38]. 


\subsection{Interpretation of the matrix ansatz as a linear mapping}

The recursion relation (34) implies that each stationary weight of the $N$-ASEP can be expressed as a linear combination of weights of the $(N-1)$-ASEP [38]. More precisely, using equation (34) we can write

$$
\left|\bar{P}^{(N)}\right\rangle=\Psi^{(N, N)}\left|\bar{P}^{(N-1)}\right\rangle,
$$

where the matrix $\Psi^{(N, N)}$ is defined in terms of its elements as

$$
\left\langle j_{1} \cdots j_{L}\left|\Psi^{(N, N)}\right| k_{1} \cdots k_{L}\right\rangle=\operatorname{Tr}\left[a_{j_{1} k_{1}}^{(N)} \cdots a_{j_{L} k_{L}}^{(N)}\right]
$$

for $1 \leq j_{i} \leq N+1$ and $1 \leq k_{i} \leq N$. Moreover the matrix $\Psi^{(N, N)}$ has the following property that we shall call sector specificity: suppose that the configuration $j_{1} \cdots j_{L}$ belongs to a basic sector $\mathfrak{s}=\left\{s_{1}<\cdots<s_{N}\right\}$ and that the element $\left\langle j_{1} \cdots j_{L}\left|\Psi^{(N, N)}\right| k_{1} \cdots k_{L}\right\rangle$ is nonzero, then the configuration $k_{1} \cdots k_{L}$ belongs to the sector $\mathfrak{s} \backslash\left\{s_{N}\right\}$. Conversely, if $j_{1} \cdots j_{L}$ belongs to $\mathfrak{s}$ and $k_{1} \cdots k_{L}$ does not belong to $\mathfrak{s} \backslash\left\{s_{N}\right\}$, then $\left\langle j_{1} \cdots j_{L}\left|\Psi^{(N, N)}\right| k_{1} \cdots k_{L}\right\rangle=0$. We will prove this property in appendix B. This property allows us to consider the mapping $\psi_{\mathfrak{s}, \mathfrak{s} \backslash\left\{s_{N}\right\}}: V_{\mathfrak{s} \backslash\left\{s_{N}\right\}} \rightarrow V_{\mathfrak{s}}$, defined as the restriction of $\Psi^{(N, N)}$ to the sectors $\mathfrak{s}$ and $\mathfrak{s} \backslash\left\{s_{N}\right\}$. This mapping provides us with a construction of the stationary state of the basic sector $\mathfrak{s}$ by lifting up that of $\mathfrak{s} \backslash\left\{s_{N}\right\}:$

$$
\left|\bar{P}_{\mathfrak{s}}\right\rangle=\psi_{\mathfrak{s}, \mathfrak{s} \backslash\left\{s_{N}\right\}}\left|\bar{P}_{\mathfrak{s} \backslash\left\{s_{N}\right\}}\right\rangle .
$$

Using this equation repeatedly, we have

$$
\left|\bar{P}_{\mathfrak{s}}\right\rangle=\psi_{\mathfrak{s}, \mathfrak{s} \backslash\left\{s_{N}\right\}} \psi_{\mathfrak{s} \backslash\left\{s_{N}\right\}, \mathfrak{s} \backslash\left\{s_{N-1}, s_{N}\right\}} \cdots \psi_{\left\{s_{1}, s_{2}\right\}\left\{s_{1}\right\}} \psi_{\left\{s_{1}\right\} \emptyset}|1 \cdots 1\rangle,
$$

where $|1 \cdots 1\rangle$ is the only configuration of the 0-TASEP on a ring of size $L$ (the configuration in which all sites are empty). Hence, the stationary state of the basic sector $\mathfrak{s}=\left\{s_{1}, \ldots, s_{N}\right\}$ is constructed along the way

$$
\emptyset \rightarrow\left\{s_{1}\right\} \rightarrow\left\{s_{1}, s_{2}\right\} \rightarrow \cdots \rightarrow \mathfrak{s} \backslash\left\{s_{N-1}, s_{N}\right\} \rightarrow \mathfrak{s} \backslash\left\{s_{N}\right\} \rightarrow \mathfrak{s}
$$

in the Hasse diagram.

On the other hand, using (23), we can project $\left|\bar{P}_{\mathfrak{s}}\right\rangle$ down to the minimal sector via arbitrary intermediate sectors, i.e. for any $\left\{n_{1}, \ldots, n_{N}\right\}=\{1, \ldots, N\}$,

$$
\varphi_{\emptyset_{\mathfrak{s}_{N-1}}} \varphi_{\mathfrak{s}_{N-1} \mathfrak{s}_{N-2}} \cdots \varphi_{\mathfrak{s}_{2} \mathfrak{s}_{1}} \varphi_{\mathfrak{s}_{1} \mathfrak{s}}\left|\bar{P}_{\mathfrak{s}}\right\rangle=\mathrm{constant}|1 \cdots 1\rangle,
$$

where $\mathfrak{s}_{x}=\mathfrak{s} \backslash\left\{s_{n_{1}}, \cdots, s_{n_{x}}\right\}$. Comparing equations (42) and (44), we observe that the $\psi$ mappings play a role opposite to that of the $\varphi$ 's. The $\psi$ 's are thus good candidates to be solutions to the conjugation relation (27). This property will be proved in the next section.

We emphasize that the matrix ansatz that we have considered above allows us to construct the stationary state only along a very specific path in the Hasse diagram: in the sector $\mathfrak{s} \backslash\left\{s_{N}\right\}$, there are $m_{1}=s_{1}$ particles of class $1, m_{2}=s_{2}-s_{1}$ particles of class $2, \ldots, m_{N-1}=s_{N-1}-s_{N-2}$ and $m_{N}=L-s_{N-1}$ particles of class $N$. In the sector $\mathfrak{s}$, the number $m_{j}$ of particles of class $j$, with $1 \leq j \leq N-1$ is the same, but there are new $m_{N}^{\prime}=s_{N}-s_{N-1}$ particles of class $N$ and $m_{N+1}^{\prime}=L-s_{N}$ particles of class $N+1$, with $m_{N+1}^{\prime}+m_{N}^{\prime}=m_{N}$. Hence, when the sector $\mathfrak{s} \backslash\left\{s_{N}\right\}$ is lifted up to $\mathfrak{s}$, a new species is created by splitting the particles of class $N$ (that have the lowest priority) into two subspecies of class $N$ and $N+1$. In the Ferrari-Martin algorithm, this means that a new species is created from the holes. However, in the Hasse diagram, there exist different paths between two connected but non-adjacent sectors. This 
observation suggests that the matrix ansatz and the Ferrari-Martin algorithm should be generalized in order to construct the stationary state via arbitrary intermediate sectors.

In the next section, we show that the matrix ansatz not only provides a way to write the stationary state but also allows one to define mappings that satisfy the conjugation relation (27) 9 . Therefore, this technique provides a tool to lift eigenvectors by intertwining the dynamics corresponding to different values of $N$. Besides, we shall also construct intertwining operators between arbitrary sectors, by defining and using more general quadratic algebras than those considered previously.

\section{Conjugation matrices from a generalized matrix ansatz}

In this section, we derive a general conjugation relation between $N$-TASEP models with different values of $N$ by using a generalized matrix ansatz which allows us to create a new class of of particles by splitting any intermediate species into two subspecies.

\subsection{Generalized quadratic algebra}

We define a family of rectangular matrices $\left\{a^{(N, n)}\right\}_{1 \leq n \leq N}$ of size $(N+1) \times N$, indexed by the integer $n$. The elements $a_{J K}^{(N, n)}=\left\langle J\left|a^{(N, n)}\right| K\right\rangle$ of the matrix $a^{(N, n)}$ are operators given in the following table:

\begin{tabular}{|c|c|c|c|}
\hline$J \backslash K$ & $1 \quad \cdots \quad n-1$ & $n$ & $n+1 \quad \cdots$ \\
\hline $\begin{array}{c}1 \\
\vdots \\
n-1\end{array}$ & $\begin{array}{c}A^{\otimes(J-1)} \otimes \delta \otimes \mathbb{1}^{\otimes(K-J-1)} \\
\otimes \epsilon \otimes \mathbb{1}^{\otimes(N-K-1)}\end{array}$ & $\begin{array}{c}A^{\otimes(J-1)} \otimes \\
\delta \otimes \mathbb{1}^{\otimes(N-J-1)}\end{array}$ & $\begin{array}{c}A^{\otimes(J-1)} \otimes \delta \otimes \mathbb{1}^{\otimes(K-J-2)} \\
\otimes \delta \otimes \mathbb{1}^{\otimes(N-K)}\end{array}$ \\
\hline$n$ & 0 & $\begin{array}{l}A^{\otimes(n-1)} \otimes \\
\mathbb{1}^{\otimes(N-n)}\end{array}$ & $\begin{array}{l}A^{\otimes(n-1)} \otimes \mathbb{1}^{\otimes(K-n-1)} \\
\quad \otimes \delta \otimes \mathbb{1}^{\otimes(N-K)}\end{array}$ \\
\hline$n+1$ & $\begin{array}{c}\mathbb{1}^{\otimes(K-1)} \otimes \epsilon \otimes \\
\mathbb{1}^{\otimes(n-K-1)} \otimes A^{\otimes(N-n)}\end{array}$ & $\begin{array}{l}\mathbb{1}^{\otimes(n-1)} \otimes \\
A^{\otimes(N-n)}\end{array}$ & 0 \\
\hline $\begin{array}{c}n+2 \\
\vdots \\
N+1\end{array}$ & $\begin{array}{c}\mathbb{1}^{\otimes(K-1)} \otimes \epsilon \otimes \mathbb{1}^{\otimes(J-K-3)} \\
\otimes \epsilon \otimes A^{\otimes(N-J+1)}\end{array}$ & $\begin{array}{c}\mathbb{1}^{\otimes(J-3)} \otimes \\
\epsilon \otimes A^{\otimes(N-J+1)}\end{array}$ & $\begin{array}{c}\mathbb{1}^{\otimes(K-2)} \otimes \delta \otimes \mathbb{1}^{\otimes(J-K-2)} \\
\otimes \epsilon \otimes A^{\otimes(N-J+1)}\end{array}$ \\
\hline
\end{tabular}

where we read $\delta \otimes \mathbb{1}^{\otimes(-1)} \otimes \epsilon=1$ and $\delta \otimes \mathbb{1}^{\otimes x} \otimes \epsilon=0$ for $x \leq-2$. The fundamental operators $\delta, \epsilon$ and $A$ satisfy the relations given in equation (36). In general, each element of $a^{(N, n)}$ is either 0 or an $(N-1)$-fold tensor product of $\mathbb{1}, A, \delta$ or $\epsilon$. Some examples are given in appendix A. Note that for the case $n=N$, one retrieves the operators that were given in the table (35).

Let us define

$$
\Psi^{(N, n)}=\operatorname{Tr}\left[\left(a^{(N, n)}\right)^{\otimes L}\right] .
$$

I This interpretation of the matrix ansatz as an intertwining operator between different dynamics was already used in a recent study of a one-species TASEP with open boundaries and with annihilation [8]. There, conjugation matrices with respect to the system size were constructed, which allowed them to calculate the normalization factor and certain correlation functions (see [49] for a related approach). 
We shall show that the following relation is satisfied:

$$
M^{(N)} \Psi^{(N, n)}=\Psi^{(N, n)} M^{(N-1)} .
$$

In other words, for any value of $n$, the matrix $\Psi^{(N, n)}$ allows us to embed the system with $(N-1)$ classes of particles into the system with $N$ classes of particles. Using $\Psi^{(N, n)}$, we shall be able to construct sector specific conjugation operators that intertwine the dynamics between any two basic sectors along any path in the Hasse diagram.

We now derive equation (47). The method used is an extension of the hat matrix technique, that was developed to prove various matrix product representations $[26,10]$. Suppose that, for each value of $n$, there exists an operator valued $(N+1) \times N$ matrix $\widehat{a}^{(N, n)}$ such that the following identity, that we shall call the hat relation, is satisfied

$$
\begin{aligned}
& M_{\mathrm{Loc}}^{(N)}\left(a^{(N, n)} \otimes a^{(N, n)}\right)-\left(a^{(N, n)} \otimes a^{(N, n)}\right) M_{\mathrm{Loc}}^{(N-1)} \\
& =a^{(N, n)} \otimes \widehat{a}^{(N, n)}-\widehat{a}^{(N, n)} \otimes a^{(N, n)} .
\end{aligned}
$$

Then, equation (47) is a consequence of this relation. Indeed, from the relation (48), we obtain, taking the $L$-fold tensor product,

$$
\begin{aligned}
& \sum_{i \in \mathbb{Z}_{L}}\left(M_{\mathrm{Loc}}^{(N)}\right)_{i, i+1}\left(a^{(N, n)}\right)^{\otimes L}-\left(a^{(N, n)}\right)^{\otimes L} \sum_{i \in \mathbb{Z}_{L}}\left(M_{\mathrm{Loc}}^{(N-1)}\right)_{i, i+1} \\
= & \sum_{i \in \mathbb{Z}_{L}}\left(a^{(N, n)}\right)^{\otimes i} \otimes \widehat{a}^{(N, n)} \otimes\left(a^{(N, n)}\right)^{\otimes(L-i-1)} \\
& -\sum_{i \in \mathbb{Z}_{L}}\left(a^{(N, n)}\right)^{\otimes(i-1)} \otimes \widehat{a}^{(N, n)} \otimes\left(a^{(N, n)}\right)^{\otimes(L-i)}=0 .
\end{aligned}
$$

Taking the trace of this relation on the space on which the operators $a^{(N, n)}$ act, and noting that local Markov matrices sum up to total Markov matrices, we obtain equation (47). We emphasize that the hat matrices $\widehat{a}^{(N, n)}$ are used in the proof but do not appear in the final result (47).

To summarize, the conjugation relation (47) follows from the hat relation (48) and in order to show that this latter relation exists, we need to specify the operators $\widehat{a}^{(N, n)}$. We claim that the hat relation (48) is satisfied for the choice

$$
\widehat{a}^{(N, n)}=d_{n} a^{(N, n)} \quad \text { with } \quad d_{n}=\operatorname{diag}(\underbrace{1, \cdots, 1}_{n}, \underbrace{0, \cdots, 0}_{N+1-n}) .
$$

This explicit expression of $\widehat{a}^{(N, n)}$ leads to closed quadratic relations between the elements $a_{J K}=\left\langle J\left|a^{(N, n)}\right| K\right\rangle(1 \leq J \leq N+1,1 \leq K \leq N)$ (Note that for simplicity, we write $a_{J K}$ instead of $\left.a_{J K}^{(N, n)}\right)$. Indeed, using the expressions of the local Markov matrices, we have

$$
\begin{aligned}
& \left\langle J J^{\prime}\left|M_{\mathrm{Loc}}^{(N)}\left(a^{(N, n)} \otimes a^{(N, n)}\right)\right| K K^{\prime}\right\rangle= \begin{cases}-a_{J K} a_{J^{\prime} K^{\prime}} & \left(J<J^{\prime}\right), \\
0 & \left(J=J^{\prime}\right), \\
a_{J^{\prime} K} a_{J K^{\prime}} & \left(J>J^{\prime}\right),\end{cases} \\
& \left\langle J J^{\prime}\left|\left(a^{(N, n)} \otimes a^{(N, n)}\right) M_{\mathrm{Loc}}^{(N-1)}\right| K K^{\prime}\right\rangle= \begin{cases}-a_{J K} a_{J^{\prime} K^{\prime}}+a_{J K^{\prime}} a_{J^{\prime} K} & \left(K<K^{\prime}\right), \\
0 & \left(K \geq K^{\prime}\right) .\end{cases}
\end{aligned}
$$

Besides, using equation (50), we can calculate each element of the right hand side of (48) as

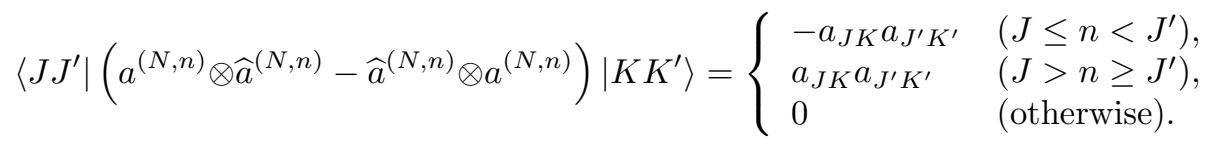


Substituting equations (51), (52) and (53) into the hat equation (48) leads to the following closed quadratic algebra generated by the operators $a_{J K}$ :

\begin{tabular}{|l|c|c|}
\hline & $\mathrm{I}\left(K<K^{\prime}\right)$ & $\mathrm{II}\left(K \geq K^{\prime}\right)$ \\
\hline $\mathrm{A}\left(J \leq n<J^{\prime}\right)$ & $a_{J K} a_{J^{\prime} K^{\prime}}=a_{J K^{\prime}} a_{J^{\prime} K}$ & - \\
\hline $\mathrm{B}\left(\begin{array}{l}J<J^{\prime} \leq n \\
\text { or } n<J<J^{\prime}\end{array}\right)$ & $a_{J K^{\prime}} a_{J^{\prime} K}=0$ & $a_{J K} a_{J^{\prime} K^{\prime}}=0$ \\
\hline $\mathrm{C}\left(J=J^{\prime}\right)$ & $a_{J K} a_{J K^{\prime}}=a_{J K^{\prime}} a_{J K}$ & - \\
\hline $\mathrm{D}\left(J>n \geq J^{\prime}\right)$ & $a_{J^{\prime} K} a_{J K^{\prime}}=a_{J K^{\prime}} a_{J^{\prime} K}$ & $a_{J K} a_{J^{\prime} K^{\prime}}=a_{J^{\prime} K} a_{J K^{\prime}}$ \\
\hline $\mathrm{E}\left(\begin{array}{l}J>J^{\prime}>n \\
\text { or } n \geq J>J^{\prime}\end{array}\right)$ & $\begin{array}{l}a_{J K} a_{J^{\prime} K^{\prime}}+a_{J^{\prime} K} a_{J K^{\prime}} \\
=a_{J K^{\prime}} a_{J^{\prime} K}\end{array}$ & $a_{J^{\prime} K} a_{J K^{\prime}}=0$ \\
\hline
\end{tabular}

(Note that the cases A-II and C-II do not give any nontrivial relation, and E-II for $K>K^{\prime}$ is equivalent to B-I.) Showing that the matrix $\Psi^{(N, n)}(46)$ satisfies the conjugation relation (47) therefore reduces to checking that $a_{J K}^{(N, n)}$ 's defined by the table (45) actually give a representation for (54). Thus the proof of the conjugation relation (47) reduces to a purely mechanical procedure. We have checked this for several values of $(N, n)$ by using Mathematica.

We emphasize that the key ingredient is the generalized hat relation (48) together with the ansatz (50) for the hat matrices $\widehat{a}^{(N, n)}$ which allows one to define a quadratic algebra. The fact that the ansatz depends on the integer $n$, with $1 \leq n \leq N$, provides a family of quadratic algebras indexed by $n$. We also note that the conjugation relation (47) shows that the quadratic algebras defined in table (54) provide representations for the stationary state of the $N$-TASEP: this is a much more concise (albeit more abstract) proof than the one given in [38].

\subsection{Sector specificity}

We consider a basic sector $\mathfrak{s}=\left\{s_{1}, \ldots, s_{N}\right\}$ and let $C(\mathfrak{s})$ be the set of all configurations of $\mathfrak{s}$. We define $\psi_{\mathfrak{s , s} \backslash\left\{s_{n^{\prime}}\right\}}^{(N, n)}: V_{\mathfrak{s} \backslash\left\{s_{n^{\prime}}\right\}} \rightarrow V_{\mathfrak{s}}$ by

$$
\left\langle j_{1} \cdots j_{L}\left|\psi_{\mathfrak{s}, \mathfrak{s} \backslash\left\{s_{n^{\prime}}\right\}}^{(N, n)}\right| k_{1} \cdots k_{L}\right\rangle=\operatorname{Tr}\left(a_{j_{1} k_{1}}^{(N, n)} \cdots a_{j_{L} k_{L}}^{(N, n)}\right)
$$

for $1 \leq n, n^{\prime} \leq N, j_{1} \cdots j_{L} \in C(\mathfrak{s})$ and $k_{1} \cdots k_{L} \in C\left(\mathfrak{s} \backslash\left\{s_{n^{\prime}}\right\}\right)$. In fact, $\psi_{\mathfrak{s}, \mathfrak{s} \backslash\left\{s_{n^{\prime}}\right\}}^{(N, n)}$ is nothing but a sub-matrix of $\Psi^{(N, n)}$. Noting that $M_{\mathfrak{s}} V_{\mathfrak{s}} \subset V_{\mathfrak{s}}$ and $M_{\mathfrak{s} \backslash\left\{s_{n^{\prime}}\right\}} V_{\mathfrak{s} \backslash\left\{s_{n^{\prime}}\right\}} \subset$ $V_{\mathfrak{s} \backslash\left\{s_{n^{\prime}}\right\}}$, we deduce from equation (47) that

$$
M_{\mathfrak{s}} \psi_{\mathfrak{s}, \mathfrak{s} \backslash\left\{s_{n^{\prime}}\right\}}^{(N, n)}=\psi_{\mathfrak{s}, \mathfrak{s} \backslash\left\{s_{n^{\prime}}\right\}}^{(N, n)} M_{\mathfrak{s} \backslash\left\{s_{n^{\prime}}\right\}},
$$

which is the conjugation relation between $\mathfrak{s}$ and $\mathfrak{s} \backslash\left\{s_{n^{\prime}}\right\}$. The following property implies that the conjugation matrix $\psi_{\mathfrak{s}, \mathfrak{s} \backslash\left\{s_{n^{\prime}}\right\}}^{(N, n)}$ vanishes unless $n^{\prime}=n$. More precisely, we have

(i) If $k_{1} \cdots k_{L}\left(1 \leq k_{\ell} \leq N\right)$ does not belong to $C\left(\mathfrak{s} \backslash\left\{s_{n}\right\}\right)$, then

$$
\operatorname{Tr}\left[a_{j_{1} k_{1}}^{(N, n)} \cdots a_{j_{L} k_{L}}^{(N, n)}\right]=0 .
$$

(ii) Equivalently,

$$
\text { if } \operatorname{Tr}\left[a_{j_{1} k_{1}}^{(N, n)} \cdots a_{j_{L} k_{L}}^{(N, n)}\right] \neq 0, \text { then } k_{1} \cdots k_{L} \in C\left(\mathfrak{s} \backslash\left\{s_{n}\right\}\right) .
$$


This statement will be proved in appendix B. Note that the sort sequences of the sectors $\mathfrak{s}$ and $\mathfrak{s} \backslash\left\{s_{n}\right\}$ can be represented as

$$
\begin{aligned}
& \overbrace{1 \cdots 1}^{s_{1}} 2 \cdots n-1 \overbrace{n \cdots n}^{s_{n}-s_{n-1}} \overbrace{n+1 \cdots n+1}^{s_{n+1}-s_{n}} n+2 \cdots \quad N \overbrace{N+1 \cdots N+1}^{L-s_{n}}, \\
& \underbrace{1 \cdots 1}_{s_{1}} 2 \cdots n-1 \underbrace{n \cdots n \quad n \quad \cdots \quad n}_{s_{n+1}-s_{n-1}} n+1 \cdots N-1 \underbrace{\begin{array}{c}
N \cdots N \\
\cdots
\end{array}}_{L-s_{n}} \text {, }
\end{aligned}
$$

respectively. The index $n$ specifies which kind of particles splits when the sector $\mathfrak{s} \backslash\left\{s_{n}\right\}$ is lifted to $\mathfrak{s}$. We write simply

$$
\psi_{\mathfrak{s}, \mathfrak{s} \backslash\left\{s_{n}\right\}}=\psi_{\mathfrak{s}, \mathfrak{s} \backslash\left\{s_{n}\right\}}^{(N, n)} .
$$

We recall that $n$ appears explicitly in (50) for the hat matrix $\widehat{a}^{(N, n)}$, leading to the quadratic algebra generated by the matrix elements of $a^{(N, n)}$. It is important to note that the sector specification property depends on the representation (45).

We emphasize that the statements $(57)$ or $(58)$ do not guarantee that $\psi_{\mathfrak{s , \mathfrak { s }} \backslash\left\{s_{n}\right\}}$ is non-vanishing (i.e. meaningful). However, based on exact calculations for small system sizes using Mathematica, we shall conjecture the stronger property that the mapping $\psi_{\mathfrak{s}, \mathfrak{s} \backslash\left\{s_{n}\right\}}$ is injective.

\subsection{Uniqueness.}

Conjugation operators $T$ from $V_{\mathfrak{s} \backslash\left\{s_{n}\right\}}$ to $V_{\mathfrak{s}}$ that satisfy $M_{\mathfrak{s}} T=T M_{\mathfrak{s} \backslash\left\{s_{n}\right\}}$ are not unique. Indeed, because of the spectral inclusion $\operatorname{Spec}(\mathfrak{s}) \supset \operatorname{Spec}\left(\mathfrak{s} \backslash\left\{s_{n}\right\}\right)$, there exist, in principle, at least $\operatorname{dim} V_{\mathfrak{s} \backslash\left\{s_{n}\right\}}$ such conjugation operators [8]. We conjecture, however, that the conjugation matrix $\psi_{\mathfrak{s}, \mathfrak{s} \backslash\left\{s_{n}\right\}}$ is unique if the following additional constraint is imposed.

Uniqueness conjecture: The solution to $M_{\mathfrak{s}} \psi_{\mathfrak{s}, \mathfrak{s} \backslash\left\{s_{n}\right\}}=\psi_{\mathfrak{s}, \mathfrak{s} \backslash\left\{s_{n}\right\}} M_{\mathfrak{s} \backslash\left\{s_{n}\right\}}$ is unique up to an overall constant factor when the following constraint is imposed:

$$
\left\langle j_{1} \cdots j_{L}\left|\psi_{\mathfrak{s}, \mathfrak{s} \backslash\left\{s_{n}\right\}}\right| k_{1} \cdots k_{L}\right\rangle=0,
$$

for all configurations $j_{1} \cdots j_{L} \in C(\mathfrak{s})$ and $k_{1} \cdots k_{L} \in C\left(\mathfrak{s} \backslash\left\{s_{n}\right\}\right)$ such that $\exists i$ such that $k_{i}+1 \leq j_{i} \leq n$ or $n+1 \leq j_{i} \leq k_{i}$.

The conjugation matrix $\psi_{\mathfrak{s}, \mathfrak{s} \backslash\left\{s_{n}\right\}}$ constructed by using the operators given in (45) satisfies this condition (see the example $(\mathrm{A} .3))^{+}$.

\subsection{Conjugation relation for $\mathfrak{s} \supset \mathfrak{t}$}

Now we turn to the construction of the conjugation matrix between Markov matrices of arbitrary sectors $\mathfrak{s}$ and $\mathfrak{t}$ such that $\mathfrak{s} \supset \mathfrak{t}$. Let us set $\mathfrak{s}=\left\{s_{1}<\cdots<s_{N}\right\}$ and $\mathfrak{s} \backslash \mathfrak{t}=\left\{s_{n_{1}}, \ldots, s_{n_{u}}\right\}$. Using the conjugation relation (56) between nearest-neighbor pairs repeatedly, we achieve the conjugation relation

$$
\psi_{\mathfrak{s t}} M_{\mathfrak{t}}=M_{\mathfrak{s}} \psi_{\mathfrak{s t}},
$$

where

$$
\psi_{\mathfrak{s t}}=\psi_{\mathfrak{s s}_{1}} \psi_{\mathfrak{s}_{1} \mathfrak{s}_{2}} \cdots \psi_{\mathfrak{s}_{u-2} \mathfrak{s}_{u-1}} \psi_{\mathfrak{s}_{u-1} \mathfrak{t}}
$$

with $\mathfrak{s}_{x}=\mathfrak{s} \backslash\left\{s_{n_{1}}, \ldots, s_{n_{x}}\right\}=\mathfrak{t} \cup\left\{s_{n_{x+1}}, \ldots, s_{n_{u}}\right\}$ (for simplicity, we have omitted the superscripts in the $\psi$ mappings as in equation (61)). Each $\psi_{\mathfrak{s}_{x-1} \mathfrak{s}_{x}}$ is constructed by

+ Note that the uniqueness conjecture does not claim that the representation (45) for the hat relation (48) is unique. 
using $a^{(N+1-x, y)}$ with $y=n_{x}-\#\left\{z \mid z<x, n_{z}<n_{x}\right\}$. A priori, this definition depends on the order chosen to enumerate the set $\mathfrak{s} \backslash \mathfrak{t}$. However, we conjecture that $\psi_{\mathfrak{s t}}$ is independent from the choice of the path in the Hasse diagram.

4.4.1. Commutativity. The path independence in the Hasse diagram can be summarized by the following statement, which we have checked for several values of $(N, n)$ by using Mathematica.

Commutativity conjecture: For any sector $\mathfrak{s}=\left\{s_{1}<\cdots<s_{N}\right\}$ and for $1 \leq x<y \leq N$,

$$
\psi_{\mathfrak{s}, \mathfrak{s} \backslash\left\{s_{x}\right\}} \psi_{\mathfrak{s} \backslash\left\{s_{x}\right\}, \mathfrak{s} \backslash\left\{s_{x}, s_{y}\right\}}=\psi_{\mathfrak{s}, \mathfrak{s} \backslash\left\{s_{y}\right\}} \psi_{\mathfrak{s} \backslash\left\{s_{y}\right\}, \mathfrak{s} \backslash\left\{s_{x}, s_{y}\right\}} .
$$

This conjecture implies that for two sectors $\mathfrak{t} \subset \mathfrak{s}$, with $\mathfrak{s} \backslash \mathfrak{t}=\left\{s_{n_{1}}, \ldots, s_{n_{u}}\right\}, \psi_{\mathfrak{s t}}$ is independent of the path in the Hasse diagram. In other words, for any reordering such that $\left\{\widetilde{n}_{1}, \ldots, \widetilde{n}_{u}\right\}=\left\{n_{1}, \ldots, n_{u}\right\}$,

$$
\psi_{\mathfrak{s}_{1}} \psi_{\mathfrak{s}_{1} \mathfrak{s}_{2}} \cdots \psi_{\mathfrak{s}_{u-2} \mathfrak{s}_{u-1}} \psi_{\mathfrak{s}_{u-1} \mathfrak{t}}=\psi_{\mathfrak{s}_{\tilde{\mathfrak{s}}_{1}}} \psi_{\tilde{\mathfrak{s}}_{1} \tilde{\mathfrak{s}}_{2}} \cdots \psi_{\tilde{\mathfrak{s}}_{u-2} \tilde{\mathfrak{s}}_{u-1}} \psi_{\tilde{\mathfrak{s}}_{u-1} \mathfrak{t}}
$$

with $\mathfrak{s}_{x}=\mathfrak{s} \backslash\left\{s_{n_{1}}, \ldots, s_{n_{x}}\right\}$ and $\tilde{\mathfrak{s}}_{x}=\mathfrak{s} \backslash\left\{s_{\tilde{n}_{1}}, \ldots, s_{\tilde{n}_{x}}\right\}$.

Remark: In particular, we note that the stationary state $\left|\bar{P}_{\mathfrak{s}}\right\rangle$ of each sector $\mathfrak{s}=\left\{s_{1}<\cdots<s_{N}\right\}$ can be obtained as

$$
\left|\bar{P}_{\mathfrak{s}}\right\rangle=\psi_{\mathfrak{s}_{1}} \psi_{\mathfrak{s}_{1} \mathfrak{s}_{2}} \cdots \psi_{\mathfrak{s}_{N-2} \mathfrak{s}_{N-1}} \psi_{\mathfrak{s}_{N-1} \emptyset}|1 \cdots 1\rangle,
$$

where we can chose the intermediate sectors $\mathfrak{s}_{x}=\left\{s_{n_{1}}, \ldots, s_{n_{x}}\right\}$ arbitrarily. Since the stationary state is unique in each sector, the compositions with different sets of intermediate sectors must be the same up to a constant factor. This observation supports the commutativity conjecture.

Example: We take $\mathfrak{s}=\{2,3,5,7,11,13\}$ and $\mathfrak{t}=\{2,7,13\}$ so that $\mathfrak{s} \backslash \mathfrak{t}=\{3,5,11\}$. If one chooses $s_{n_{1}}=11, s_{n_{2}}=5$ and $s_{n_{3}}=3$, then the intermediate conjugation matrices are constructed from $a^{(6,5)}, a^{(5,3)}$ and $a^{(4,2)}$, respectively, and this leads to the conjugation matrix $\psi_{\mathfrak{s t}}$

$$
\psi_{\mathfrak{s t}}=\psi_{\{2,3,5,7,11,13\},\{2,3,5,7,13\}} \psi_{\{2,3,5,7,13\},\{2,3,7,13\}} \psi_{\{2,3,7,13\},\{2,7,13\}} \text {. }
$$

Taking $s_{n_{1}}=5, s_{n_{2}}=3$ and $s_{n_{3}}=11$, we obtain the following conjugation matrix:

$$
\widetilde{\psi}_{\mathfrak{s t}}=\psi_{\{2,3,5,7,11,13\},\{2,3,7,11,13\}} \psi_{\{2,3,7,11,13\},\{2,7,11,13\}} \psi_{\{2,7,11,13\},\{2,7,13\}},
$$

where the intermediate conjugation matrices are constructed by $a^{(6,3)}, a^{(5,2)}$ and $a^{(4,3)}$, respectively. The commutativity conjecture implies that $\psi_{\mathfrak{s t}}=\widetilde{\psi}_{\mathfrak{s t}}$.

\subsection{Generalized Ferrari-Martin algorithm}

The algebraic construction that we have presented for all values of $n$, with $1 \leq n \leq N$, can be turned into an algorithm to calculate the stationary weights of the $N$-TASEP, that generalizes the original Ferrari-Martin algorithm [24]. Figure 4 provides an example for $(N, n)=(5,3)$.

Given two sectors $\mathfrak{s}=\left\{s_{1}<\ldots<s_{N}\right\}$ and $\mathfrak{s} \backslash\left\{s_{n}\right\}$, the matrix product representation of the conjugation matrix $\psi_{\mathfrak{s}, \mathfrak{s} \backslash\left\{s_{n}\right\}}$ leads to the following generalization of the Ferrari-Marin algorithm. 
(ii)

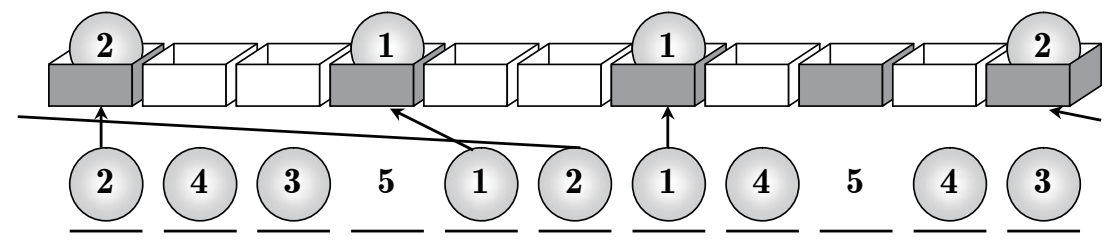

(iii)

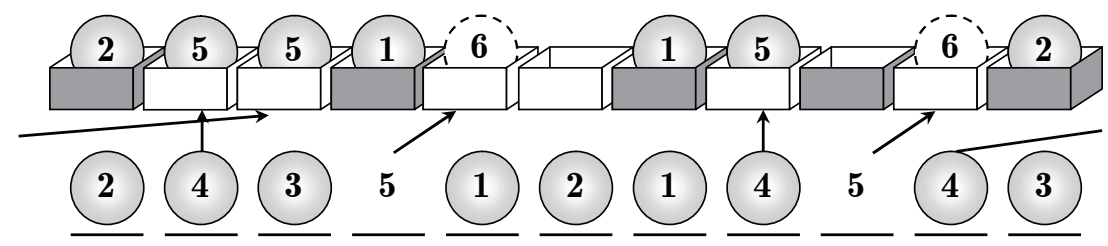

(iv)

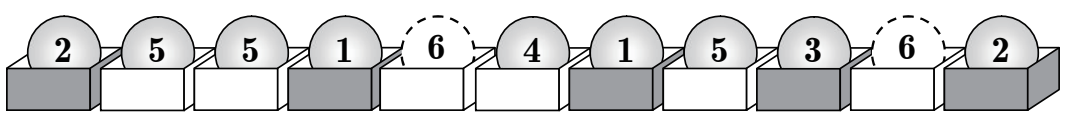

Figure 4. The generalized Ferrari-Martin algorithm that constructs a configuration of $N$-TASEP from that of $(N-1)$-TASEP. This figure provides the example $F($ bwwbwwbwbwb,24351214543) $=25516415362$.

(i) Set $s_{n}$ black boxes and $\left(L-s_{n}\right)$ white boxes arbitrarily as $c_{1} \cdots c_{L}\left(c_{i}=b, w\right)$ on the upper line, and a configuration $k_{1} \cdots k_{L}$ of the sector $\mathfrak{s} \backslash\left\{s_{n}\right\}$ on the lower line. There are

$$
\begin{aligned}
& \left(s_{\nu}-s_{\nu-1}\right) \nu \text { th-class particles } \quad\left(\text { for } 1 \leq \nu \leq n-1, s_{0}=0\right), \\
& \left(s_{n+1}-s_{n-1}\right) n \text { th-class particles, } \\
& \left(s_{\nu+1}-s_{\nu}\right) \nu \text { th-class particles } \quad\left(\text { for } n+1 \leq \nu \leq N, s_{N+1}=L\right)
\end{aligned}
$$

on the lower line.

As in the original Ferrari-Martin algorithm, put particles of $\nu$ th class from $\nu=1$ to $\nu=n-1$, according to the rule (ii- $\nu$ ).

(ii- $\nu)$ There are $\left(s_{n}-s_{\nu-1}\right)$ unoccupied black boxes. Let $\left\{i_{1}, \ldots, i_{s_{\nu}-s_{\nu-1}}\right\}$ be the positions of the $\nu$ th-class particles. Iterate the following procedure $\left(s_{\nu}-s_{\nu-1}\right)$ times; find the nearest unoccupied black box $c_{i^{\prime}}=b$ with $i^{\prime} \leq i_{\ell}$ for the $\ell$ th $\nu\left(=k_{i_{\ell}}\right)$, and put the particle $\nu$ on it or on the rightmost unoccupied black box if $i^{\prime}$ does not exist.

Put particles of $(\nu+1)$ th class from $\nu=N$ to $\nu=n+1$, according to the rule (iii- $\nu$ ) which is opposite to (ii).

(iii- $\nu)$ There are $\left(s_{\nu+1}-s_{n}\right)$ unoccupied white boxes. Let $\left\{i_{1}, \ldots, i_{s_{\nu+1}-s_{\nu}}\right\}$ be the positions of the $\nu$ th-class particles. Iterate the following procedure $\left(s_{\nu+1}-s_{\nu}\right)$ times; find the nearest unoccupied white box $c_{i^{\prime}}=w$ with $i^{\prime} \geq i_{\ell}$ for the $\ell$ th $\nu\left(=k_{i_{\ell}}\right)$, and put the particle $\nu+1$ on it or on the leftmost unoccupied white box if $i^{\prime}$ does not exist. (Note that for $\nu=N$, we "put empty sites" (i.e., particles of $(N+1)$ th class). $)$ 
(iv) There are $\left(s_{n}-s_{n-1}\right)$ unoccupied black boxes and $\left(s_{n+1}-s_{n}\right)$ unoccupied white boxes. Put $n$ 's and $(n+1)$ 's on them, respectively. We have a configuration $F\left(c_{1} \cdots c_{L}, k_{1} \cdots k_{L}\right)$ on the upper line.

This generalized Ferrari-Martin algorithm constructs the same conjugation matrix:

$$
\psi_{\mathfrak{s}, \mathfrak{s} \backslash\left\{s_{n}\right\}}=\sum\left|F\left(c_{1} \cdots c_{L}, k_{1} \cdots k_{L}\right)\right\rangle\left\langle k_{1} \cdots k_{L}\right|,
$$

where $\sum$ runs over $c_{1} \cdots c_{L}$ and $k_{1} \cdots k_{L}$ with $\#\left\{i \mid c_{i}=b\right\}=s_{n}$ and $k_{1} \cdots k_{L}$ belonging to the sector $\mathfrak{s} \backslash\left\{s_{n}\right\}$.

This algorithm allows us to close the loop between this work and the previous articles $[24,18,38]$. In these previous works, the original Ferrari-Martin algorithm, in which empty sites played a very special role, was used to construct a quadratic algebra to represent the stationary state. Here, we have found a family of matrix product representations that allow to split any given species into two subspecies (so that empty sites do not play a distinguished role anymore).

To conclude this section, we precisely show the equivalence (73) between the matrix product representation and the generalized Ferrari-Martin algorithm (GFMA). We first note that each nonzero element of $a_{J K}^{(N, n)}(45)$ has the form

$$
a_{J K}^{(N, n)}=a_{J K, 1}^{(N, n)} \otimes \cdots \otimes a_{J K, n-1}^{(N, n)} \otimes a_{J K, n+1}^{(N, n)} \otimes \cdots \otimes a_{J K, N}^{(N, n)} \text { with } a_{J K, \nu}^{(N, n)} \in\{\mathbb{1}, A, \delta, \epsilon\} .
$$

(Note the shift in the subscripts that occurs for $\nu>n$ ). Let

$$
\left.\mathcal{A}=\bigoplus_{\mu \geq 0} \mathbb{C}|\mu\rangle\right\rangle,\left\langle\langle\mu|=\left(0, \ldots, \underset{(\mu+1) \mathrm{th}}{0,1,0, \ldots),|\mu\rangle\rangle=\left\langle\left\langle\left.\mu\right|^{\mathrm{T}}\right.\right.}\right.\right.
$$

be the space on which the matrix $a_{J K, \nu}^{(N, n)}$ acts (thus $a_{J K}^{(N, n)}$ acts on $\mathcal{A}^{\otimes(N-1)}$ ). The fundamental matrices $A, \delta$ and $\epsilon$ act on $|\mu\rangle\rangle$ as

$$
\left.A|\mu\rangle\rangle=\left\{\begin{array}{ll}
0 & (\mu \geq 1), \\
|0\rangle\rangle & (\mu=0),
\end{array} \quad \delta|\mu\rangle\right\rangle=\left\{\begin{array}{ll}
|\mu-1\rangle\rangle & (\mu \geq 1), \\
0 & (\mu=0),
\end{array} \quad \epsilon|\mu\rangle\right\rangle=|\mu+1\rangle\right\rangle .
$$

The form (74) implies that its trace is again decomposed as

$\operatorname{Tr}\left(a_{j_{1} k_{1}}^{(N, n)} \cdots a_{j_{L} k_{L}}^{(N, n)}\right)=\left\{\begin{array}{l}\prod_{\substack{1 \leq \nu \leq N \\ \nu \neq n}} \operatorname{Tr}\left(a_{j_{1} k_{1}, \nu}^{(N, n)} \cdots a_{j_{L} k_{L}, \nu}^{(N, n)}\right) \quad\left(\text { every } a_{j_{i} k_{i}}^{(N, n)} \neq 0\right), \\ 0 \quad\left(\text { at least one } a_{j_{i} k_{i}}^{(N, n)}=0\right) .\end{array}\right.$

Furthermore, we find

$$
\begin{aligned}
& \prod_{\substack{1 \leq \nu \leq N \\
\nu \neq n}} \operatorname{Tr}\left(a_{j_{1} k_{1}, \nu}^{(N, n)} \cdots a_{j_{L} k_{L}, \nu}^{(N, n)}\right)=\prod_{\substack{1 \leq \nu \leq N \\
\nu \neq n}} \sum_{\mu_{\nu}^{\prime} \geq 0}\left\langle\left\langle\mu_{\nu}^{\prime}\left|a_{j_{1} k_{1}, \nu}^{(N, n)} \cdots a_{j_{L} k_{L}, \nu}^{(N, n)}\right| \mu_{\nu}^{\prime}\right\rangle\right\rangle \\
& = \begin{cases}\prod_{\substack{1 \leq \nu \leq N \\
\nu \neq n}}\left\langle\left\langle\mu_{\nu}\left|a_{j_{1} k_{1}, \nu}^{(N, n)} \cdots a_{j_{L} k_{L}, \nu}^{(N, n)}\right| \mu_{\nu}\right\rangle\right\rangle=1 & (*), \\
0 & \text { (otherwise), }\end{cases}
\end{aligned}
$$

The symbol $*$ denotes the case where there exists $\left(\mu_{1}, \ldots \mu_{n-1}, \mu_{n+1}, \ldots, \mu_{N}\right)$ such that

$$
\left.\left.\left.a_{j_{1} k_{1}, \nu}^{(N, n)} \cdots a_{j_{L} k_{L}, \nu}^{(N, n)}\left|\mu_{\nu}\right\rangle\right\rangle=\left|\mu_{\nu}\right\rangle\right\rangle, \quad a_{j_{1} k_{1}, \nu}^{(N, n)} \cdots a_{j_{L} k_{L}, \nu}^{(N, n)}\left|\mu_{\nu}^{\prime}\right\rangle\right\rangle=0\left(\mu_{\nu}^{\prime} \neq \mu_{\nu}\right) .
$$

The set of numbers $\left(\mu_{1}, \ldots \mu_{n-1}, \mu_{n+1}, \ldots, \mu_{N}\right)$ which satisfies this condition is unique if it exists. This uniqueness is true for basic sectors. On the other hand, we draw a 
vertical line at each bond in the GFMA as in figure 5 . The correspondence between the algorithm and the action of the operators can be understood by regarding $|\mu\rangle\rangle$ as the number $\mu$ of arrows $\nu \rightarrow \nu(\nu<n)$ or $\nu \rightarrow \nu+1(\nu>n)$ crossing each vertical line: $\delta$ decreases the number $\mu$ of arrows, $\epsilon$ increases $\mu, A$ tests whether $\mu=0$ or not, and the identity operator $\mathbb{1}$ indeed does nothing. Then we find that, for given configurations $j_{1} \cdots j_{L}$ and $k_{1} \cdots k_{L}$ of $N$-species and $(N-1)$-species sectors, the matrix product $a_{j_{1} k_{1}}^{(N, n)} \cdots a_{j_{L} k_{L}}^{(N, n)}$ gives a unique pattern of arrows (if $*$ is satisfied). This means that $j_{1} \cdots j_{L}$ can be obtained by using the GFMA from $k_{1} \cdots k_{L}$ :

$$
F\left(c_{1} \cdots c_{L}, k_{1} \cdots k_{L}\right)=j_{1} \cdots j_{L} \quad \text { with } \quad c_{i}=b\left(j_{i} \leq n\right), w\left(j_{i} \geq n+1\right) .
$$

This relation is true because the representation (45) obeys the rule of the GFMA. (For instance, the $\nu$ th element of $a_{j_{i} k_{i}}^{(N, n)}$ for $j_{i}, k_{i}<n$ and $\nu \leq j_{i}-1$ is $A$, which means that $j_{i}$ can be put on the $i$ th site of the upper line only when no arrow $\nu \rightarrow \nu$ crosses the vertical line between sites $i$ and $i+1$.) Thus, this graphical construction shows that the matrix product representation and the GFMA are euivalent.

Figure 5 provides an example of the correspondence in the case $(N, n)=(3,2)$ : for $j_{1} \cdots j_{10}=3211414433$ and $k_{1} \cdots k_{10}=1233321212$, the actions of $a_{j_{i} k_{i}, 1}^{(3,2)}$ and $a_{j_{i} k_{i}, 3}^{(3,2)}$ give trajectories of the numbers of arrows $1 \rightarrow 1$ and $3 \rightarrow 4$, respectively. Namely, the condition $*$ is satisfied for these configurations: one can show

$$
\begin{aligned}
& \left.\left.\left.a_{j_{1} k_{1}, 1}^{(3,2)} \cdots a_{j_{10} k_{10}, 1}^{(3,2)}|1\rangle\right\rangle=|1\rangle\right\rangle, \quad a_{j_{1} k_{1}, 1}^{(3,2)} \cdots a_{j_{10} k_{10}, 1}^{(3,2)}\left|\mu_{1}^{\prime}\right\rangle\right\rangle=0\left(\mu_{1}^{\prime} \neq 1\right), \\
& \left.\left.\left.a_{j_{1} k_{1}, 3}^{(3,2)} \cdots a_{j_{10} k_{10}, 3}^{(3,2)}|0\rangle\right\rangle=|0\rangle\right\rangle, \quad a_{j_{1} k_{1}, 3}^{(3,2)} \cdots a_{j_{10} k_{10}, 3}^{(3,2)}\left|\mu_{3}^{\prime}\right\rangle\right\rangle=0\left(\mu_{3}^{\prime} \neq 0\right) .
\end{aligned}
$$

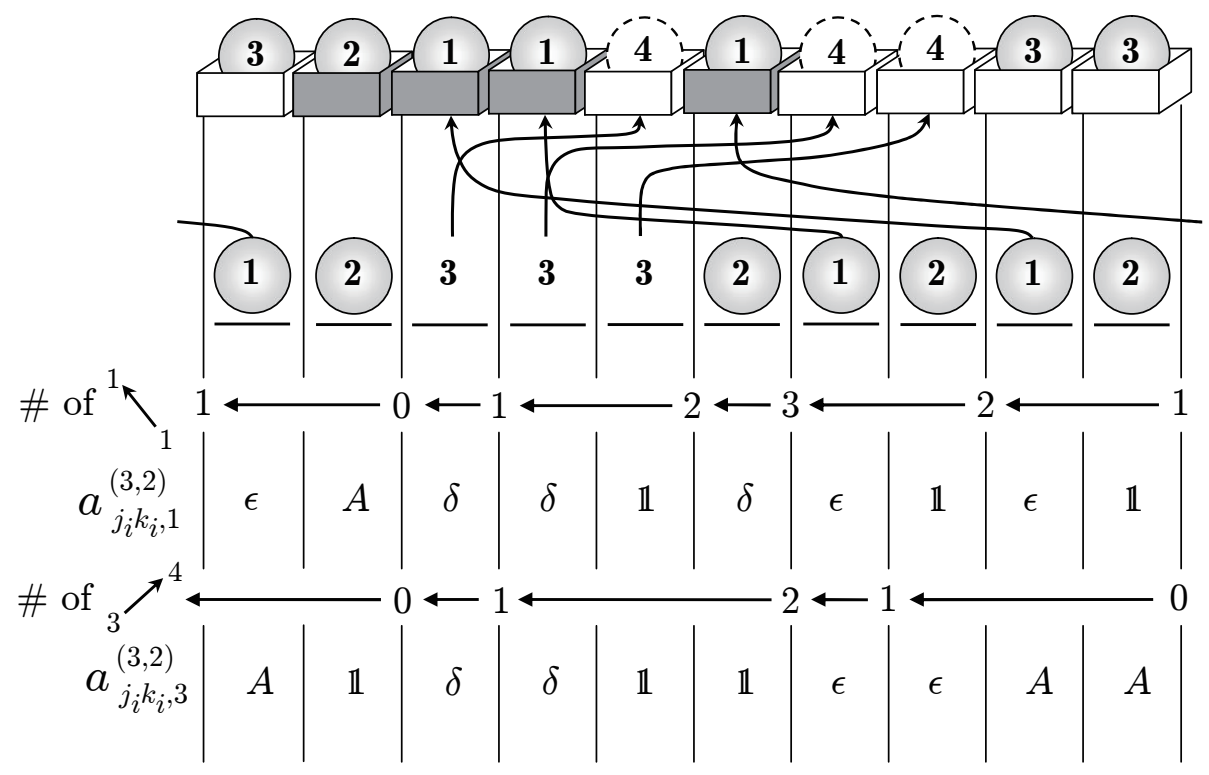

Figure 5. Correspondence between the representation (45) and the generalized Ferrari-Martin algorithm. 


\section{Concluding remarks}

The multispecies exclusion process exhibits rich combinatorial properties that can be encoded in a recursive structure known as the Hasse diagram. The relevance of this diagram can be understood by using identification maps: if one blurs the difference between particles that belong to two adjacent classes, a simpler system is obtained that inherits the properties of the original system. Successive identifications allow one to reduce the initial multispecies system to the one-species system. In this procedure, information is irreversibly lost.

In this paper, we have shown that the matrix product representation, which was originally used as a method for representing the stationary state, allows one to define conjugation operators that relate systems with different values of $N$ in the TASEP case. Therefore, the matrix ansatz provides a method to lift information from a simple system to a more complex one: it allows one to calculate not only the stationary state but some excited states as well, that describe how the system relaxes towards its steady state. More precisely, each link in the Hasse diagram corresponds to a lifting operator and is associated to a different quadratic algebra. Hence, the $N$-TASEP leads to families of algebras, connected through compatibility relations. We believe that this feature is general and that the investigation initiated here could be extended in the following directions:

(i) The $N$-TASEP is an integrable model and its Markov matrix can be viewed one special member of a family of commuting transfer matrices for the Perk-Schultz model $[39,42]$. One natural question is to study if the matrix product representation for such vertex models allows one to define conjugation operators.

(ii) We also emphasize that we have studied here the $N$-TASEP only, where particles hop in one definite direction. It is natural to expect that our results could be extended to the $N$-PASEP. A naive guess would be to start with the TASEP solution (45) and make the following replacements

$$
\delta \rightarrow \delta_{q}, \epsilon \rightarrow \epsilon_{q}, A \rightarrow A_{q}
$$

where $\delta_{q}, \epsilon_{q}$ and $A_{q}$ satisfy the $q$-deformed quadratic relations (38). However, this guess is correct only for $n=1$ and $n=N$ but wrong for $1<n<N$. The basic mathematical reason is that the $q$-deformed quadratic relations (38) are not stable by tensor product unless $q=0$ : a different approach seems to be required for solving the $N$-PASEP in full generality.

\section{Acknowledgments}

This work is supported by Grant-in-Aid for Young Scientists ((B) 22740106) and Global COE Program "Education and Research Hub for Mathematics-for-Industry".

\section{Appendix A. An explicit example}

Here we write down the operator-valued rectangular matrix $a^{(N, n)}(45)$ for a few values of $(N, n)$.

$$
a^{(2,1)}=\begin{aligned}
& 1 \\
& 2 \\
& 3
\end{aligned}\left(\begin{array}{ll}
1 & 2 \\
A & 0 \\
\epsilon & \mathbb{1}
\end{array}\right), \quad a^{(2,2)}=\begin{array}{r}
1 \\
2 \\
3
\end{array}\left(\begin{array}{ll}
1 & 2 \\
0 & \delta \\
0 & A \\
\epsilon & \mathbb{1}
\end{array}\right),
$$




$$
\begin{aligned}
& a^{(3,1)}=\quad a^{(3,2)}=\quad a^{(3,3)}=
\end{aligned}
$$

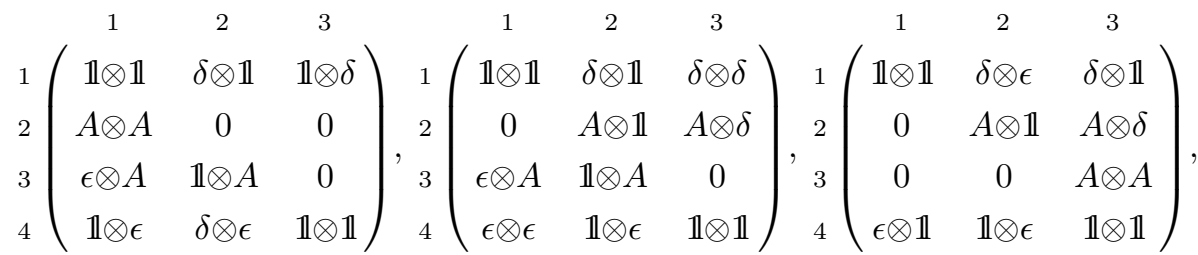

$$
\begin{aligned}
& a^{(6,3)}= \\
& \begin{array}{llllll}
1 & 2 & 3 & 4 & 5 & 6
\end{array}
\end{aligned}
$$

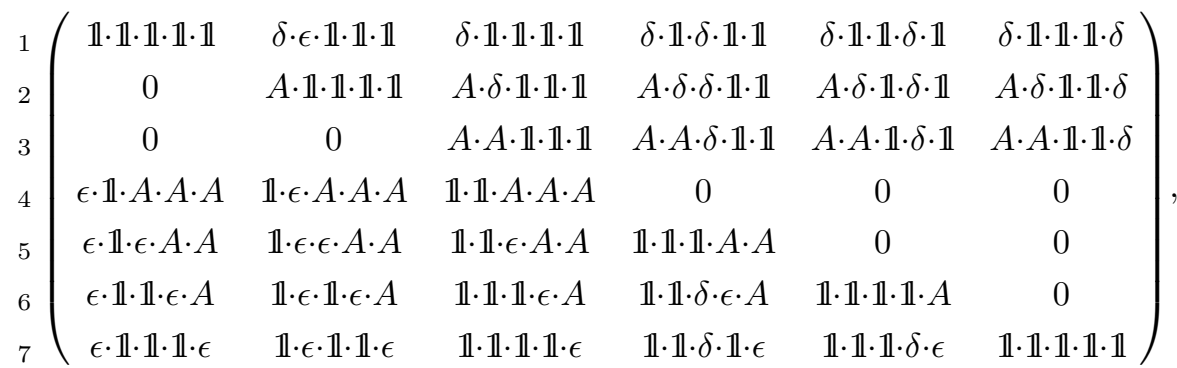

where we replaced $\otimes$ by $\cdot$. We also give an example for the new conjugation matrix $\psi$ as well as the identification $\varphi$ for $\{1,2,3\}$ and $\{1,3\}((N, n)=(3,2))$ with $L=4$ (the entries of the matrices equal to 0 are replaced by · for better readability):

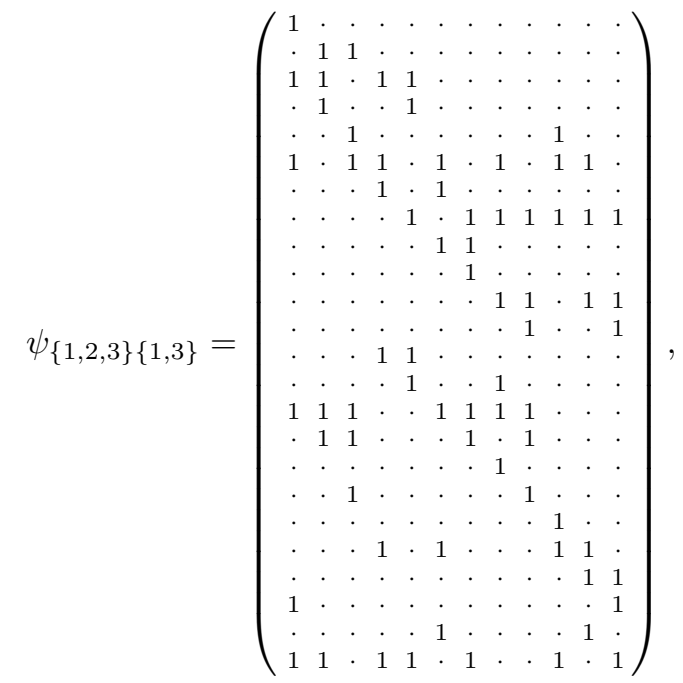

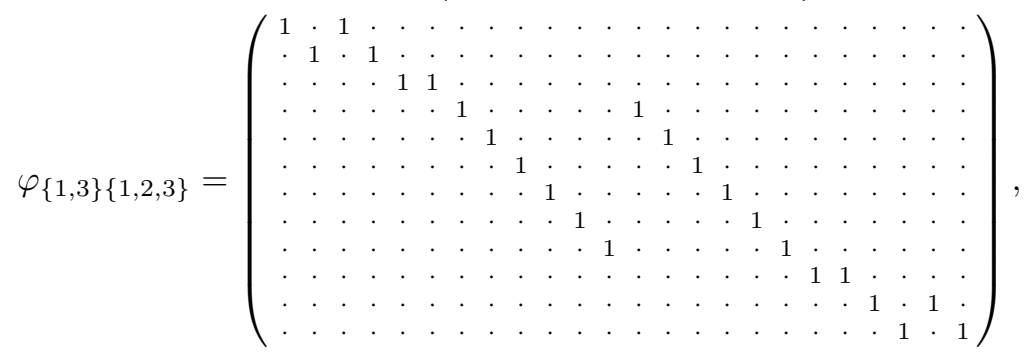


where the bases are arranged as $1234,1243, \ldots, 4321$ for $\{1,2,3\}$, and $1223,1232, \ldots, 3221$ for $\{1,3\}$. They satisfy

$$
\begin{aligned}
& \varphi_{\{1,3\}\{1,2,3\}} M_{\{1,2,3\}}=M_{\{1,3\}} \varphi_{\{1,3\}\{1,2,3\}}, \\
& \psi_{\{1,2,3\}\{1,3\}} M_{\{1,3\}}=M_{\{1,2,3\}} \psi_{\{1,2,3\}\{1,3\}} .
\end{aligned}
$$

The Markov matrix $M_{\{1,3\}}$ has eigenvalue $E=-1$, and we write its corresponding eigenvector $|E\rangle$ :

$$
|E\rangle=|1223\rangle-|1322\rangle+|2132\rangle-|2213\rangle-|2231\rangle+|2312\rangle-|3122\rangle+|3221\rangle
$$

The conjugation matrix $\psi_{\{1,2,3\}\{1,3\}}$ lifts $|E\rangle$ to the sector $\{1,2,3\}$ as

$$
\begin{aligned}
& \left|E^{\prime}\right\rangle:=\psi_{\{1,2,3\}\{1,3\}}|E\rangle= \\
& |1234\rangle-|1243\rangle+2|1324\rangle+|1342\rangle-2|1423\rangle-|1432\rangle-|2134\rangle+|2143\rangle \\
& -2|2314\rangle-|2341\rangle+2|2413\rangle+|2431\rangle+|3124\rangle+2|3142\rangle-|3214\rangle-2|3241\rangle \\
& +|3412\rangle-|3421\rangle-|4123\rangle-2|4132\rangle+|4213\rangle+2|4231\rangle-|4312\rangle+|4321\rangle
\end{aligned}
$$

The vector $\left|E^{\prime}\right\rangle$ is an eigenvector of $M_{\{1,2,3\}}\left(M_{\{1,2,3\}}\left|E^{\prime}\right\rangle=E\left|E^{\prime}\right\rangle\right)$. The identification operator reconstructs the eigenvector in the sector $\{1,3\}$ :

$$
\varphi_{\{1,3\}\{1,2,3\}}\left|E^{\prime}\right\rangle=3|E\rangle \text {. }
$$

\section{Appendix B. Proof of the statement (58)}

We shall use the following property: let $b_{i} \in\{\mathbb{1}, A, \delta, \epsilon\}(1 \leq i \leq L)$.

$$
\#\left\{i \mid b_{i}=\delta\right\} \neq \#\left\{i \mid b_{i}=\epsilon\right\} \Rightarrow \operatorname{Tr}\left(b_{1} \cdots b_{L}\right)=0 .
$$

We also note the decomposition (77).

We first consider the case $(N, n)=(3,2)$ as an example. Noting the properties (B.1) and (77), we find the following necessary condition such that $\operatorname{Tr}\left(a_{j_{1} k_{1}}^{(3,2)} \cdots a_{j_{L} k_{L}}^{(3,2)}\right) \neq 0$ : every $a_{j_{i} k_{i}}^{(3,2)}$ is nonzero and

$$
\#\left\{i \mid a_{j_{i} k_{i}, \nu}^{(3,2)}=\delta\right\}=\#\left\{i \mid a_{j_{i} k_{i}, \nu}^{(3,2)}=\epsilon\right\}
$$

for $\nu=1,3$. For given 3- and 2-species configurations $j_{1} \cdots j_{L}$ and $k_{1} \cdots k_{L}$, we have

$$
\begin{aligned}
& \#\left\{i \mid j_{i}=1\right\}=\#\left\{i \mid a_{j_{i} k_{i}, 1}^{(3,2)}=\delta\right\}+\#\left\{i \mid j_{i}=k_{i}=1\right\}, \\
& \#\left\{i \mid j_{i}=4\right\}=\#\left\{i \mid a_{j_{i} k_{i}, 3}^{(3,2)}=\epsilon\right\}+\#\left\{i \mid j_{i}=k_{i}+1=4\right\}, \\
& \#\left\{i \mid k_{i}=1\right\}=\#\left\{i \mid a_{j_{i} k_{i}, 1}^{(3,2)}=\epsilon\right\}+\#\left\{i \mid j_{i}=k_{i}=1\right\}, \\
& \#\left\{i \mid k_{i}=3\right\}=\#\left\{i \mid a_{j_{i} k_{i}, 3}^{(3,2)}=\delta\right\}+\#\left\{i \mid j_{i}-1=k_{i}=3\right\},
\end{aligned}
$$

assuming that every $a_{j_{i} k_{i}}^{(3,2)} \neq 0$. (See the explicit form for $a^{(3,2)}$ (A.2).) The condition (B.2) and the relations (B.3)-(B.6) imply that

$$
\#\left\{i \mid j_{i}=1\right\}=\#\left\{i \mid k_{i}=1\right\}, \quad \#\left\{i \mid j_{i}=4\right\}=\#\left\{i \mid k_{i}=3\right\} .
$$

This consequence means that if $j_{1} \cdots j_{L} \in C\left(\left\{s_{1}, s_{2}, s_{3}\right\}\right)$ and $\operatorname{Tr}\left(a_{j_{1} k_{1}}^{(3,2)} \cdots a_{j_{L} k_{L}}^{(3,2)}\right) \neq 0$ then $k_{1} \cdots k_{L} \in C\left(\left\{s_{1}, s_{3}\right\}\right)$. 
In the GFMA, the condition (B.2) corresponds to the conservation of the number of arrows when going around the ring (80). As in figure 5 , for $j_{1} \cdots j_{L}=3211414433$ and $k_{1} \cdots k_{L}=1233321212$, we actually observe

$$
\begin{aligned}
& \#\left\{i \mid a_{j_{i} k_{i}, 1}^{(3,2)}=\delta\right\}=\#\left\{i \mid a_{j_{i} k_{i}, 1}^{(3,2)}=\epsilon\right\}=3, \\
& \#\left\{i \mid a_{j_{i} k_{i}, 3}^{(3,2)}=\delta\right\}=\#\left\{i \mid a_{j_{i} k_{i}, 3}^{(3,2)}=\epsilon\right\}=2 .
\end{aligned}
$$

For the general case, a necessary condition for $\operatorname{Tr}\left(a_{j_{1} k_{1}}^{(N, n)} \cdots a_{j_{L} k_{L}}^{(N, n)}\right) \neq 0$ is that every $a_{j_{i} k_{i}}^{(N, n)}$ is nonzero and

$$
\#\left\{i \mid a_{j_{i} k_{i}, \nu}^{(N, n)}=\delta\right\}=\#\left\{i \mid a_{j_{i} k_{i}, \nu}^{(N, n)}=\epsilon\right\} \text { for } \forall \nu \in\{1, \ldots, n-1, n+1, \ldots, N\} .
$$

We can assume that every $a_{j_{i} k_{i}}^{(N, n)}$ is nonzero. From the definition (45), we find

$$
\begin{aligned}
& \#\left\{i \mid j_{i}=\nu\right\}= \\
& \left\{\begin{array}{cl}
\#\left\{i \mid a_{j_{i} k_{i}, \nu}^{(N, n)}=\delta\right\}+\#\left\{i \mid j_{i}=k_{i}=\nu\right\} & (1 \leq \nu \leq n-1), \\
\#\left\{i \mid a_{j_{i} k_{i}, \nu-1}^{(N, n)}=\epsilon\right\}+\#\left\{i \mid j_{i}=k_{i}+1=\nu\right\} & (n+2 \leq \nu \leq N+1),
\end{array}\right. \\
& \#\left\{i \mid k_{i}=\nu\right\}= \\
& \begin{cases}\#\left\{i \mid a_{j_{i} k_{i}, \nu}^{(N, n)}=\epsilon\right\}+\#\left\{i \mid j_{i}=k_{i}=\nu\right\} & (1 \leq \nu \leq n-1), \\
\#\left\{i \mid a_{j_{i} k_{i}, \nu}^{(N, n)}=\delta\right\}+\#\left\{i \mid j_{i}-1=k_{i}=\nu\right\} & (n+1 \leq \nu \leq N) .\end{cases}
\end{aligned}
$$

Consequently, we have

$$
\begin{aligned}
& \#\left\{i \mid j_{i}=\nu\right\}=\#\left\{i \mid k_{i}=\nu\right\} \quad \text { for } 1 \leq \nu \leq n-1, \\
& \#\left\{i \mid j_{i}=\nu\right\}=\#\left\{i \mid k_{i}=\nu-1\right\} \quad \text { for } n+2 \leq \nu \leq N+1,
\end{aligned}
$$

which exactly means that if $j_{1} \cdots j_{L} \in C(\mathfrak{s}) \quad\left(\mathfrak{s}=\left\{s_{1}, \ldots, s_{N}\right\}\right)$ and $\operatorname{Tr}\left(a_{j_{1} k_{1}}^{(N, n)} \cdots a_{j_{L} k_{L}}^{(N, n)}\right) \neq 0$, then $k_{1} \cdots k_{L} \in \mathfrak{s} \backslash\left\{s_{n}\right\}$. (See the figures of the sort sequences (59) and (60)). We emphasize, however, that even if $j_{1} \cdots j_{L} \in C(\mathfrak{s})$ and $k_{1} \cdots k_{L} \in C\left(\mathfrak{s} \backslash\left\{s_{n}\right\}\right)$, the matrix element $\operatorname{Tr}\left[a_{j_{1} k_{1}}^{(N, n)} \cdots a_{j_{L} k_{L}}^{(N, n)}\right]$ can still be equal to zero. 
[1] F. C. Alcaraz and R. Z. Bariev, Exact solution of asymmetric diffusion with N classes of particles of arbitrary size and hierarchical order, Braz. J. Phys. 30, 655 (2000).

[2] F. C. Alcaraz and V. Rittenberg, Reaction-diffusion processes as physical realizations of Hecke algebras Phys. Lett. B 314, 377 (1993).

[3] F. J. Alexander, S.A. Janowski, J. L. Lebowitz and H. Van Beijeren, Shock fluctuations in onedimensional lattice fluids, Phys. Rev. E 47, 403 (1993).

[4] E. D. Andjel, M. Bramson and T. M. Liggett, Shocks in the asymmetric simple exclusion process, Prob. Theor. Rel. Fields 78, 231 (1988).

[5] O. Angel, The stationary measure of a 2-type totally asymmetric exclusion process, J. Comb. Theory A 113, 625 (2006).

[6] C. Arita, A. Kuniba, K. Sakai and T. Sawabe, Spectrum of a multi-species asymmetric simple exclusion process on a ring, J. Phys. A 42, 345002 (2009).

[7] P. F. Arndt, T. Heinzel and V. Rittenberg, Spontaneous breaking of translational invariance in one-dimensional stationary states on a ring, J. Phys. A 31, L45 (1998).

[8] A. Ayyer and K. Mallick, Exact results for an asymmetric annihilation process with open boundaries, J. Phys. A 43, 045003 (2010).

[9] R. J. Baxter, Exactly solved models in statistical mechanics, Dover (2007).

[10] R. A. Blythe and M. R. Evans, Nonequilibrium steady states of matrix product form: A solver's guide, J. Phys. A 40, R333 (2007).

[11] C. Boldrighini, G. Cosimi, S. Frigio and M. Grasso Nuñes, Computer simulation of shock waves in the completely asymmetric simple exclusion process, J. Stat. Phys. 55, 611 (1989).

[12] S. Chatterjee and M. Barma, Shock probes in a one-dimensional Katz-Lebowitz-Spohn model, Phys. Rev. E 77, 061124 (2008).

[13] T. Chou and D. Lohse, Entropy-driven pumping in zeolites and biological channels, Phys. Rev. Lett. 82, 3552 (1999).

[14] B. Derrida, An exactly soluble non-equilibrium system: the asymmetric simple exclusion process, Phys. Rep. 301, 65 (1998).

[15] B. Derrida, M. R. Evans, V. Hakim and V. Pasquier, An exact solution of a 1D asymmetric exclusion model using a matrix formulation J. Phys. A 26, 1493 (1993).

[16] B. Derrida, S.A. Janowski, J. L. Lebowitz and E. R. Speer, Exact solution of the totally asymmetric exclusion process: shock profiles, J. Stat. Phys. 73, 813 (1993).

[17] E. Duchi and G. Schaeffer, A combinatorial approach to jumping particles, J. Comb. Theory A 110, $1(2005)$

[18] M. R. Evans, P. A. Ferrari and K. Mallick, Matrix Representation of the Stationary Measure for the Multispecies TASEP, J. Stat. Phys. 135, 217 (2009).

[19] M.R. Evans, Y. Kafri, H.M. Koduvely and D. Mukamel, Phase separation and coarsening in one-dimensional driven diffusive systems: Local dynamics leading to long-range Hamiltonians Phys. Rev. E 58, 2764 (1998).

[20] P. A. Ferrari, Shock fluctuations in the asymmetric simple exclusion, Prob. Theor. Rel. Fields 91, 81 (1992).

[21] P. A. Ferrari, L. R. G. Fontes, Current fluctuations for the asymmetric exclusion process, Ann. Prob. 22, 1 (1994).

[22] P. A. Ferrari, L. R. G. Fontes and Y. Kohayakawa, Invariant measures for a two-species asymmetric process, J. Stat. Phys. 76, 1153 (1994).

[23] P. A. Ferrari and J. B. Martin, Multiclass processes, dual points and M/M/1 queues, Markov Proc. Rel. Fields 12, 175 (2006).

[24] P. A. Ferrari and J. B. Martin, Stationary distributions of multi-type totally asymmetric exclusion processes, Ann. Prob. 35, 807 (2007).

[25] T. Halpin-Healy, Y.-C. Zhang, Kinetic roughening phenomena, stochastic growth, directed polymers and all that, Phys. Rep. 254, 215 (1995).

[26] H. Hinrichsen, I. Peschel, S. Sandow, On Matrix Product Ground States for Reaction-Diffusion Models, J. Phys. A 29, 2643 (1996).

[27] S. A. Janowski, J. L. Lebowitz, Finite size effects and Shock fluctuations in the asymmetric exclusion process, Phys. Rev. A 45, 618 (1992).

[28] V. Karimipour, Multispecies asymmetric simple exclusion process and its relation to traffic flow, Phys. Rev. E 59, 205 (1999).

[29] S. Katz, J. L. Lebowitz and H. Spohn, Nonequilibrium steady states of stochastic lattice gas models of fast ionic conductors, J. Stat. Phys. 34, 497 (1984).

[30] S. Klumpp, T. M. Nieuwenhuizen and R. Lipowsky, Movements of molecular motors: Ratchets, random walks and traffic phenomena, Physica E 29, 380 (2005).

[31] J. L. Lebowitz, E. Presutti and H. Spohn, Microscopic models of hydrodynamic behaviour, J. 
Stat. Phys. 51, 841 (1988).

[32] D. G. Levitt, Dynamics of a single-file pore: non-fickian behavior, Phys. Rev. A 8, 3050 (1973).

[33] T. M. Liggett, Coupling the Simple Exclusion Process, Ann. Probab. 4, 339 (1976).

[34] T. M. Liggett, Interacting Particle Systems, Springer-Verlag New-York, (1985).

[35] T. M. Liggett, Stochastic Models of Interacting Systems:Contact, Voter and Exclusion Processes, Springer-Verlag New-York, (1999).

[36] C. T. MacDonald, J. H. Gibbs, A. C. Pipkin, 1968, Kinetics of biopolymerization on nucleic acid templates, Biopolymers 6, 1 (1968).

[37] K. Mallick, S. Mallick and N. Rajewski, Exact solution of an exclusion process with three classes of particles and vacancies, J. Phys. A 32, 8399 (1999).

[38] S. Prolhac, M. R. Evans, K. Mallick, The matrix product solution of the multispecies partially asymmetric exclusion process, J. Phys. A 42, 165004 (2009).

[39] J. H. H. Perk and C. L. Schultz, New families of commuting transfer matrices in q-state vertex models, Phys. Lett. 84A, 407 (1981).

[40] A. Schadschneider, Statistical mechanics of traffic flow, Physica A 285, 101 (2001).

[41] B. Schmittmann and R. K. P. Zia, Statistical mechanics of driven diffusive systems, in Phase Transitions and Critical Phenomena vol 17., C. Domb and J. L. Lebowitz Ed., Academic Press San Diego, (1995).

[42] C. L. Schultz, Eigenvectors of the Multi-Component Generalization of the six-vertex model, Physica 122A, 71 (1983).

[43] G. M. Schütz, Exactly Solvable Models for Many-Body Systems Far from Equilibrium in Phase Transitions and Critical Phenomena vol 19., C. Domb and J. L. Lebowitz Ed., Academic Press San Diego, (2001).

[44] F. Spitzer, Interaction of Markov Processes, Adv. in Math. 5, 246 (1970).

[45] H. Spohn, Large scale dynamics of interacting particles, Springer-Verlag New-York, (1991).

[46] R. P. Stanley, Enumerative combinatorics, vol. 1, Cambridge Univ. Press (2000).

[47] B. Sutherland, Beautiful Models: 70 Years of Exactly Solved Quantum Many-Body Problems, World Scient. Pub. Comp. (2004).

[48] S. R. S. Varadhan, The complex story of simple exclusion in In itô stochastic calculus and probability theory, N. Ikeda, S. Watanabe, M. Fukushima and H. Kunita Eds., Springer (1996).

[49] M. Woelki and K. Mallick, Transfer matrices for the totally asymmetric simple exclusion process, J. Phys. A 43, 185003 (2010). 


\section{List of MI Preprint Series, Kyushu University}

The Global COE Program

Math-for-Industry Education \& Research Hub

MI

MI2008-1 Takahiro ITO, Shuichi INOKUCHI \& Yoshihiro MIZOGUCHI

Abstract collision systems simulated by cellular automata

MI2008-2 Eiji ONODERA

The intial value problem for a third-order dispersive flow into compact almost

Hermitian manifolds

MI2008-3 Hiroaki KIDO

On isosceles sets in the 4-dimensional Euclidean space

MI2008-4 Hirofumi NOTSU

Numerical computations of cavity flow problems by a pressure stabilized characteristiccurve finite element scheme

MI2008-5 Yoshiyasu OZEKI

Torsion points of abelian varieties with values in nfinite extensions over a padic field

MI2008-6 Yoshiyuki TOMIYAMA

Lifting Galois representations over arbitrary number fields

MI2008-7 Takehiro HIROTSU \& Setsuo TANIGUCHI

The random walk model revisited

MI2008-8 Silvia GANDY, Masaaki KANNO, Hirokazu ANAI \& Kazuhiro YOKOYAMA Optimizing a particular real root of a polynomial by a special cylindrical algebraic decomposition

MI2008-9 Kazufumi KIMOTO, Sho MATSUMOTO \& Masato WAKAYAMA

Alpha-determinant cyclic modules and Jacobi polynomials 
MI2008-10 Sangyeol LEE \& Hiroki MASUDA

Jarque-Bera Normality Test for the Driving Lévy Process of a Discretely Observed Univariate SDE

MI2008-11 Hiroyuki CHIHARA \& Eiji ONODERA

A third order dispersive flow for closed curves into almost Hermitian manifolds

MI2008-12 Takehiko KINOSHITA, Kouji HASHIMOTO and Mitsuhiro T. NAKAO

On the $L^{2}$ a priori error estimates to the finite element solution of elliptic problems with singular adjoint operator

MI2008-13 Jacques FARAUT and Masato WAKAYAMA

Hermitian symmetric spaces of tube type and multivariate Meixner-Pollaczek polynomials

MI2008-14 Takashi NAKAMURA

Riemann zeta-values, Euler polynomials and the best constant of Sobolev inequality

MI2008-15 Takashi NAKAMURA

Some topics related to Hurwitz-Lerch zeta functions

MI2009-1 Yasuhide FUKUMOTO

Global time evolution of viscous vortex rings

MI2009-2 Hidetoshi MATSUI \& Sadanori KONISHI

Regularized functional regression modeling for functional response and predictors

MI2009-3 Hidetoshi MATSUI \& Sadanori KONISHI

Variable selection for functional regression model via the $L_{1}$ regularization

MI2009-4 Shuichi KAWANO \& Sadanori KONISHI

Nonlinear logistic discrimination via regularized Gaussian basis expansions

MI2009-5 Toshiro HIRANOUCHI \& Yuichiro TAGUCHII

Flat modules and Groebner bases over truncated discrete valuation rings 
MI2009-6 Kenji KAJIWARA \& Yasuhiro OHTA

Bilinearization and Casorati determinant solutions to non-autonomous $1+1$ dimensional discrete soliton equations

\section{MI2009-7 Yoshiyuki KAGEI}

Asymptotic behavior of solutions of the compressible Navier-Stokes equation around the plane Couette flow

MI2009-8 Shohei TATEISHI, Hidetoshi MATSUI \& Sadanori KONISHI

Nonlinear regression modeling via the lasso-type regularization

MI2009-9 Takeshi TAKAISHI \& Masato KIMURA

Phase field model for mode III crack growth in two dimensional elasticity

MI2009-10 Shingo SAITO

Generalisation of Mack's formula for claims reserving with arbitrary exponents for the variance assumption

MI2009-11 Kenji KAJIWARA, Masanobu KANEKO, Atsushi NOBE \& Teruhisa TSUDA Ultradiscretization of a solvable two-dimensional chaotic map associated with the Hesse cubic curve

\section{MI2009-12 Tetsu MASUDA}

Hypergeometric T -functions of the q-Painlevé system of type $E_{8}^{(1)}$

MI2009-13 Hidenao IWANE, Hitoshi YANAMI, Hirokazu ANAI \& Kazuhiro YOKOYAMA A Practical Implementation of a Symbolic-Numeric Cylindrical Algebraic Decomposition for Quantifier Elimination

MI2009-14 Yasunori MAEKAWA

On Gaussian decay estimates of solutions to some linear elliptic equations and its applications

MI2009-15 Yuya ISHIHARA \& Yoshiyuki KAGEI

Large time behavior of the semigroup on $L^{p}$ spaces associated with the linearized compressible Navier-Stokes equation in a cylindrical domain 
MI2009-16 Chikashi ARITA, Atsuo KUNIBA, Kazumitsu SAKAI \& Tsuyoshi SAWABE Spectrum in multi-species asymmetric simple exclusion process on a ring

MI2009-17 Masato WAKAYAMA \& Keitaro YAMAMOTO

Non-linear algebraic differential equations satisfied by certain family of elliptic functions

MI2009-18 Me Me NAING \& Yasuhide FUKUMOTO

Local Instability of an Elliptical Flow Subjected to a Coriolis Force

MI2009-19 Mitsunori KAYANO \& Sadanori KONISHI

Sparse functional principal component analysis via regularized basis expansions and its application

MI2009-20 Shuichi KAWANO \& Sadanori KONISHI

Semi-supervised logistic discrimination via regularized Gaussian basis expansions

MI2009-21 Hiroshi YOSHIDA, Yoshihiro MIWA \& Masanobu KANEKO

Elliptic curves and Fibonacci numbers arising from Lindenmayer system with symbolic computations

MI2009-22 Eiji ONODERA

A remark on the global existence of a third order dispersive flow into locally Hermitian symmetric spaces

MI2009-23 Stjepan LUGOMER \& Yasuhide FUKUMOTO

Generation of ribbons, helicoids and complex scherk surface in laser-matter Interactions

MI2009-24 Yu KAWAKAMI

Recent progress in value distribution of the hyperbolic Gauss map

MI2009-25 Takehiko KINOSHITA \& Mitsuhiro T. NAKAO

On very accurate enclosure of the optimal constant in the a priori error estimates for $H_{0}^{2}$-projection 
MI2009-26 Manabu YOSHIDA

Ramification of local fields and Fontaine's property (Pm)

MI2009-27 Yu KAWAKAMI

Value distribution of the hyperbolic Gauss maps for flat fronts in hyperbolic three-space

MI2009-28 Masahisa TABATA

Numerical simulation of fluid movement in an hourglass by an energy-stable finite element scheme

MI2009-29 Yoshiyuki KAGEI \& Yasunori MAEKAWA Asymptotic behaviors of solutions to evolution equations in the presence of translation and scaling invariance

MI2009-30 Yoshiyuki KAGEI \& Yasunori MAEKAWA

On asymptotic behaviors of solutions to parabolic systems modelling chemotaxis

MI2009-31 Masato WAKAYAMA \& Yoshinori YAMASAKI

Hecke's zeros and higher depth determinants

MI2009-32 Olivier PIRONNEAU \& Masahisa TABATA

Stability and convergence of a Galerkin-characteristics finite element scheme of lumped mass type

MI2009-33 Chikashi ARITA

Queueing process with excluded-volume effect

MI2009-34 Kenji KAJIWARA, Nobutaka NAKAZONO \& Teruhisa TSUDA

Projective reduction of the discrete Painlevé system of type $\left(A_{2}+A_{1}\right)^{(1)}$

MI2009-35 Yosuke MIZUYAMA, Takamasa SHINDE, Masahisa TABATA \& Daisuke TAGAMI Finite element computation for scattering problems of micro-hologram using DtN map 
MI2009-36 Reiichiro KAWAI \& Hiroki MASUDA

Exact simulation of finite variation tempered stable Ornstein-Uhlenbeck processes

MI2009-37 Hiroki MASUDA

On statistical aspects in calibrating a geometric skewed stable asset price model

MI2010-1 Hiroki MASUDA

Approximate self-weighted LAD estimation of discretely observed ergodic OrnsteinUhlenbeck processes

MI2010-2 Reiichiro KAWAI \& Hiroki MASUDA

Infinite variation tempered stable Ornstein-Uhlenbeck processes with discrete observations

MI2010-3 Kei HIROSE, Shuichi KAWANO, Daisuke MIIKE \& Sadanori KONISHI Hyper-parameter selection in Bayesian structural equation models

MI2010-4 Nobuyuki IKEDA \& Setsuo TANIGUCHI

The Itô-Nisio theorem, quadratic Wiener functionals, and 1-solitons

MI2010-5 Shohei TATEISHI \& Sadanori KONISHI

Nonlinear regression modeling and detecting change point via the relevance vector machine

MI2010-6 Shuichi KAWANO, Toshihiro MISUMI \& Sadanori KONISHI

Semi-supervised logistic discrimination via graph-based regularization

MI2010-7 Teruhisa TSUDA

UC hierarchy and monodromy preserving deformation

MI2010-8 Takahiro ITO

Abstract collision systems on groups

MI2010-9 Hiroshi YOSHIDA, Kinji KIMURA, Naoki YOSHIDA, Junko TANAKA \& Yoshihiro MIWA

An algebraic approach to underdetermined experiments 
MI2010-10 Kei HIROSE \& Sadanori KONISHI

Variable selection via the grouped weighted lasso for factor analysis models

MI2010-11 Katsusuke NABESHIMA \& Hiroshi YOSHIDA

Derivation of specific conditions with Comprehensive Groebner Systems

MI2010-12 Yoshiyuki KAGEI, Yu NAGAFUCHI \& Takeshi SUDOU

Decay estimates on solutions of the linearized compressible Navier-Stokes equation around a Poiseuille type flow

MI2010-13 Reiichiro KAWAI \& Hiroki MASUDA

On simulation of tempered stable random variates

MI2010-14 Yoshiyasu OZEKI

Non-existence of certain Galois representations with a uniform tame inertia weight

MI2010-15 Me Me NAING \& Yasuhide FUKUMOTO

Local Instability of a Rotating Flow Driven by Precession of Arbitrary Frequency

MI2010-16 Yu KAWAKAMI \& Daisuke NAKAJO

The value distribution of the Gauss map of improper affine spheres

MI2010-17 Kazunori YASUTAKE

On the classification of rank 2 almost Fano bundles on projective space

MI2010-18 Toshimitsu TAKAESU

Scaling limits for the system of semi-relativistic particles coupled to a scalar bose field

MI2010-19 Reiichiro KAWAI \& Hiroki MASUDA

Local asymptotic normality for normal inverse Gaussian Lévy processes with high-frequency sampling

MI2010-20 Yasuhide FUKUMOTO, Makoto HIROTA \& Youichi MIE

Lagrangian approach to weakly nonlinear stability of an elliptical flow 
MI2010-21 Hiroki MASUDA

Approximate quadratic estimating function for discretely observed Lévy driven SDEs with application to a noise normality test

MI2010-22 Toshimitsu TAKAESU

A Generalized Scaling Limit and its Application to the Semi-Relativistic Particles System Coupled to a Bose Field with Removing Ultraviolet Cutoffs

MI2010-23 Takahiro ITO, Mitsuhiko FUJIO, Shuichi INOKUCHI \& Yoshihiro MIZOGUCHI Composition, union and division of cellular automata on groups

MI2010-24 Toshimitsu TAKAESU

A Hardy's Uncertainty Principle Lemma in Weak Commutation Relations of Heisenberg-Lie Algebra

MI2010-25 Toshimitsu TAKAESU

On the Essential Self-Adjointness of Anti-Commutative Operators

MI2010-26 Reiichiro KAWAI \& Hiroki MASUDA

On the local asymptotic behavior of the likelihood function for Meixner Lévy processes under high-frequency sampling

MI2010-27 Chikashi ARITA \& Daichi YANAGISAWA

Exclusive Queueing Process with Discrete Time

MI2010-28 Jun-ichi INOGUCHI, Kenji KAJIWARA, Nozomu MATSUURA \& Yasuhiro OHTA

Motion and Bäcklund transformations of discrete plane curves

MI2010-29 Takanori YASUDA, Masaya YASUDA, Takeshi SHIMOYAMA \& Jun KOGURE On the Number of the Pairing-friendly Curves

MI2010-30 Chikashi ARITA \& Kohei MOTEGI

Spin-spin correlation functions of the $q$-VBS state of an integer spin model

MI2010-31 Shohei TATEISHI \& Sadanori KONISHI

Nonlinear regression modeling and spike detection via Gaussian basis expansions 
MI2010-32 Nobutaka NAKAZONO

Hypergeometric $\tau$ functions of the $q$-Painlevé systems of type $\left(A_{2}+A_{1}\right)^{(1)}$

MI2010-33 Yoshiyuki KAGEI

Global existence of solutions to the compressible Navier-Stokes equation around parallel flows

MI2010-34 Nobushige KUROKAWA, Masato WAKAYAMA \& Yoshinori YAMASAKI

Milnor-Selberg zeta functions and zeta regularizations

MI2010-35 Kissani PERERA \& Yoshihiro MIZOGUCHI

Laplacian energy of directed graphs and minimizing maximum outdegree algorithms

MI2010-36 Takanori YASUDA

CAP representations of inner forms of $S p(4)$ with respect to Klingen parabolic subgroup

MI2010-37 Chikashi ARITA \& Andreas SCHADSCHNEIDER

Dynamical analysis of the exclusive queueing process

MI2011-1 Yasuhide FUKUMOTO\& Alexander B. SAMOKHIN

Singular electromagnetic modes in an anisotropic medium

MI2011-2 Hiroki KONDO, Shingo SAITO \& Setsuo TANIGUCHI

Asymptotic tail dependence of the normal copula

MI2011-3 Takehiro HIROTSU, Hiroki KONDO, Shingo SAITO, Takuya SATO, Tatsushi TANAKA \& Setsuo TANIGUCHI

Anderson-Darling test and the Malliavin calculus

MI2011-4 Hiroshi INOUE, Shohei TATEISHI \& Sadanori KONISHI

Nonlinear regression modeling via Compressed Sensing

MI2011-5 Hiroshi INOUE

Implications in Compressed Sensing and the Restricted Isometry Property

MI2011-6 Daeju KIM \& Sadanori KONISHI

Predictive information criterion for nonlinear regression model based on basis expansion methods

MI2011-7 Shohei TATEISHI, Chiaki KINJYO \& Sadanori KONISHI

Group variable selection via relevance vector machine 
MI2011-8 Jan BREZINA \& Yoshiyuki KAGEI

Decay properties of solutions to the linearized compressible Navier-Stokes equation around time-periodic parallel flow

Group variable selection via relevance vector machine

MI2011-9 Chikashi ARITA, Arvind AYYER, Kirone MALLICK \& Sylvain PROLHAC Recursive structures in the multispecies TASEP 